\title{
Extension of the standard model of electroweak interaction and Dark Matter in the tangent bundle geometry
}

\author{
Joachim Herrmann ${ }^{\text {a }}$ \\ Max Born Institute, Max Born Straße 2a, 12489 Berlin, Germany
}

Received: 8 February 2019 / Accepted: 1 September 2019 / Published online: 20 September 2019

(C) The Author(s) 2019

\begin{abstract}
A generalized theory of electroweak interaction is developed based on the underlying geometrical structure of the tangent bundle with symmetries arising from transformations of tangent vectors along the fiber axis at a fixed spacetime point given by the $\mathrm{SO}(3,1)$ group. Electroweak interaction beyond the standard model (SM) is described by the little groups $S U(2) \otimes E^{c}(2)$ ( $E^{c}(2)$ is the central extended Euclidian group) which includes the group $S U(2) \otimes U(1)$ as a limit case. In addition to isospin and hypercharge, two additional quantum numbers arise which explain the existence of families in the SM. The connection coefficients yield the SM gauge potentials but also hypothetical gauge bosons and other hypothetical particles as a Higgs family as well as candidate Dark Matter particles are predicted. Several important consequences for the interaction between dark fermions, dark scalars or dark vector gauge bosons with each other and with SM Higgs and Z-bosons are described.
\end{abstract}

\section{Introduction}

The formal equivalence of gauge theories with the geometry of fiber bundles has been recognized since the 1960s [1-5]. In the fiber bundle formalism, gauge potentials are understood as a geometrical entity - the connections on the principal bundles, and matter fields are described by associated fiber bundles. The geometrical interpretation of gauge theories by the mathematical fiber bundle theory is a beautiful and mathematically profound concept. However, in earlier investigations the transformation groups of the fibers were taken from the phenomenologically determined internal gauge groups of the Standard Model (SM). Therefore, up to now the fiber bundle interpretation yields mainly a re-interpretation of the gauge fields and did not effectuated a physical theory beyond the SM.

\footnotetext{
a e-mail: jherrman@mbi-berlin.de
}

In this paper we consider the general hypothesis that the fundamental physical interactions can be described within the geometrical structure of the most fundamental fiber bundle - the tangent bundle, and gauge transformations can be identified with transformations at a fixed spacetime point along the tangent vector axis leaving the scalar product invariant. This means the gauge group is not assumed for phenomenological reasons but is taken to arise self-consistently from the invariance of the scalar product with respect to tangent fiber transformations described by the group $S O(3,1)$. Since the action of this group is not transitive, the vector space decomposes into different orbits and the most general (projective) irreducible representations of $\mathrm{SO}(3,1)$ can be found by the little groups $S U(2), E^{c}(2)$ and $S U(1,1)$ where the group $E^{c}(2)$ is the central extended Euclidean group. Based on differential geometry on the tangent bundle with covariant derivatives determined by the generators of the transformation group $G=S U(2) \otimes E^{c}(2)$ and corresponding connection coefficients (gauge potentials) a generalized theory of the electroweak interaction is derived. In addition to the internal quantum numbers (IQN) of isospin and hypercharge, the $E^{c}$ charge $\varkappa$ and the family quantum number $n$ arise which could elucidate the existence of families in the SM. In this approach the known $Z$ and $W^{ \pm}$gauge bosons can be found again, but in addition new extra $E^{c}$ and $B^{ \pm}$gauge bosons and other hypothetical particles as e.g. a family of Higgs particles are predicted. A notable feature of the theory presented is the possibility of identifying candidate stable or unstable hypothetical Dark Matter (DM) vector bosons, DM scalars and DM fermions with zero hypercharge and zero isospin but nonzero $E^{c}$-charge $\varkappa \neq 0$ without additional phenomenological model assumptions. Here we present only the basics, specific in-depth observable consequences are beyond the scope of the present paper. Note that the more general transformation group $S O(3,1) \rtimes T(3,1)$ (where $T(3,1)$ is the translational group and $\rtimes$ represents the semi-direct product) includes teleparallel gravity into the tangent bundle geometry based 
on translational transformations $T(3,1)$ of the tangent fibers. According to this approach the interpretation of the $S O(3,1)$ connection coefficients as electroweak gauge potentials is compatible with teleparallel gauge gravity theory, which is fully equivalent to Einstein's general relativity theory.

\section{Differential geometry on the tangent bundle}

We start with a brief description of the geometry of the tangent bundle on a manifold (see e.g. [6]). The tangent space $T_{x}(M)$ at the point $x$ on the spacetime manifold $M$ is the set of all tangent vectors spanned by frame vectors in the coordinate basis $e_{\mu}=\partial_{\mu}(\mu=0,1,2,3)$. The tangent bundle is the union of all tangent spaces at all points $x$ of the manifold $M: T M=\bigcup_{x \in M} T_{x}(M)$. In a coordinate description a point in $T M$ is described by the numbers of pairs $X=(x, u)$ with $x=\left\{x^{0}, x^{1}, x^{2}, x^{3}\right)$ as the coordinates of the spacetime manifold and $u=\left\{u^{0}, u^{1}, u^{2}, u^{3}\right)$ are the coordinates of the tangent vectors. Thus the tangent fiber bundle geometry introduces four additional variables $u$ for the description of the tangent fiber. To aid understanding, it is convenient to consider $M$ as a pseudo-Riemannian spacetime manifold with indefinite metric $g^{\mu \nu}(x)$. Besides the frame vectors in the coordinate basis $e_{\mu}=\partial_{\mu}(\mu=0,1,2,3)$, one can introduce the tetrads as another geometric object on the tangent space:

$e_{a}=e_{a}^{\mu}(x) \partial_{\mu}$.

Each vector described in the coordinate basis $e_{\mu}=\partial_{\mu}$ can be expressed by a vector with respect to the tetrad frame basis $e_{a}$ according to the rule

$v^{v}=e_{a}^{v}(x) v^{a}$.

The subscripts $a, b, \ldots$ number the vectors $(a, b=0,1,2,3)$ and $\mu$ their components in the coordinate basis. The dual basis of the frame fields $e_{a}$ are cotangent frame 1-forms $e^{a}=$ $e_{\mu}^{a} d x^{\mu}$ satisfying the orthogonality relation $e_{a}^{\mu}(x) e_{v}^{a}(x)=$ $\delta_{v}^{\mu}$. By using the tetrads of the pseudo-Riemannian manifold, the scalar product of two vectors is given by the Lorentz metric:

$$
\begin{aligned}
(v, u) & =g_{\mu \nu}(x) v^{\mu} u^{v}=g_{\mu \nu}(x) e_{a}^{\mu}(x) e_{b}^{v}(x) v^{a} u^{b} \\
& =\eta_{a b} v^{a} u^{b}
\end{aligned}
$$

where $\eta_{a b}=\operatorname{diag}(-1,1,1,1)$ is the metric of the Minkovski space.

The geometric properties of manifolds are usually related to the invariance of certain geometrical structure relations under the action of certain transformation groups. The definition of the scalar product (3) is the governing structure relation defining the geometry of the tangent bundle. Tangent vectors manifest two kinds of transformations which do not change the scalar product in (3). Under general coordinate transformations of the spacetime manifold $x^{\mu} \rightarrow y^{\mu}=$ $y^{\mu}(x)$ vectors transform as $v^{\prime \mu}(x)=\left(\partial y^{\mu} / \partial x^{v}\right) v^{v}(x)$. On the other hand, the vector components in the tetrad frame basis remain unchanged: $v^{\prime a}(x)=v^{a}$. A second type of transformations exists that does not change the scalar product. These are transformations at a fixed point $x$ of the spacetime manifold $M$ transforming the tangent vectors along the tangent fiber directions as follows:

$v^{\prime a}=T_{b}^{a}(x) v^{b}, e_{\mu}^{a}=\left(T_{b}^{a}(x)\right) e_{\mu}^{b}$,

$e_{a}^{\mu}(x)=\left(T_{a}^{b}(x)\right)^{-1} e_{b}^{\mu}(x)$,

where $T_{b}^{a}(x)$ are matrices satisfying the conditions $\eta_{a b} T_{c}^{a} T_{d}^{b}=\eta_{c d}$. On the other hand, the tangent vectors which refer to the coordinate frame remain unchanged: $v^{\prime \mu}=v^{\mu}$. The transformation group of tangent vectors along the tangent fiber is the $S O(3,1)$ group of special linear transformations, with matrix elements $T_{b}^{a}(x) \in S O(3,1)$ depending on the spacetime point $x$ as a parameter.

Note that the transformation of the tangent vectors by the group $S O(3,1)$ is not the most general transformation. Actually, the fact that the group $S O(3,1)$ leaves the scalar product of tangent vectors invariant is not sufficient because we need the infinitesimal tangent vector line elements to be invariant. We have

$(\mathrm{d} v, \mathrm{~d} u)=g_{\mu \nu}(x) \mathrm{d} v^{\mu} \mathrm{d} u^{v}=\eta_{a b} \mathrm{~d} v^{a} \mathrm{~d} u^{b}$.

This allows us to add constant translations to the transformations in (4):

$v^{\prime a}=T_{b}^{a}(x) v^{b}+a^{a}(x)$,

and this leads to the more general transformation group $S O(3,1) \rtimes T(3,1)$.

Poincaré transformations and the transformation group (6) of tangent vectors $T_{b}^{a}(x)$ in the tetrad basis are described by the same group $S O(3,1) \rtimes T(3,1)$ but the two have a principally different geometrical and physical meaning: the former transforms the spacetime coordinates of a flat manifold while the latter describes transformations within the tangent fiber $F=T_{x}(M)$ leaving the spacetime point $x$ unchanged. The fact that Poincaré transformations (defined as coordinate transformations of spacetime in a flat manifold) and the transformation (6) are based on the same mathematical group could lead to confusion, which can be avoided if the principally different meaning of the two transformations is taken into account. As an example, the Coleman-Mandula theorem [7] states that the combination of spacetime symmetries with internal symmetries is not possible in any but the trivial way. And this is what here is the case, the group $S O(3,1)$ in (4) or $S O(3,1) \rtimes T(3,1)$ in $(6)$ are the internal groups and they 
are combined with spacetime transformations as explained below (3) only in a trivial way.

\section{Connections on the tangent bundle and teleparallel gauge gravity theory}

The geometric construction of tetrads is closely linked to the conceptional basis of gravity theories and its extensions to gravity gauge theories. To facilitate a proper understanding of the underlying geometric structure and a unified description including gravity we first consider the general inhomogeneous transformation group $S O(3,1) \rtimes T(3,1)$ and its relationship with gravity. The differential geometry on the tangent bundle can be obtained using the general rules for principal fiber bundles $P(M ; G)$ requiring the definition of connections and covariant derivatives on the bundle. The definition of a covariant derivative demands considering vectors which point from one fiber to the other at different points $x$ and $x^{\prime}$ of the spacetime manifold. The generators $\mathbf{L}_{a}$ of the group $G$ are vertical vectors pointing along the fiber and therefore belong to the vertical subspace $V_{u}(P)$. Horizontal vectors in the subspace $H_{u}(P)$ which point away from the fibers (i.e. elements of the tangent space of the fiber bundle $T_{u}(P)$ that complement the vertical vectors in $V_{u}(P)$ ) can be constructed by the definition of a connection as an assignment to each point in the principal fiber such that $[8,9]$

$T_{u}(P)=H_{u}(P) \oplus V_{u}(P)$.

The definition of a connection can be used for the definition of a covariant differentiation along the curves horizontally lifted to the principal bundle:

$\frac{\mathrm{d}}{\mathrm{d} \tau}=\frac{\mathrm{d} x^{\mu}}{\mathrm{d} \tau} D_{\mu}$,

where

$D_{\mu}=\frac{\partial}{\partial x^{\mu}}+i \widetilde{A_{\mu}^{a}} \mathbf{L}_{a}$

is the covariant derivative on the principal fiber bundle. The $\mathbf{L}_{a}$ are the right-invariant fundamental vector fields (generators) on the group manifold $G=\left\{g_{i j}\right\}$ and $\widetilde{A_{\mu}^{a}}$ the connection coefficients of the group $G$. A connection on a principal bundle induces a connection on the associated bundle. The covariant derivative on the associated bundle is given by (9) substituting the generators $\mathbf{L}_{a}$ by the left-invariant fundamental vector fields on the section of the associated bundle, which describe matter fields.

The geometric transformations of tangent vectors in a tangent bundle are described by the group $G=S O(3,1) \rtimes$ $T(3,1)$. According to (9) the covariant derivative along the horizontal lifted curve on the principal bundle $P(M ; G)$ of this group is given by
$D_{\mu}=\frac{\partial}{\partial x^{\mu}}+i \omega_{. \mu}^{a} \mathbf{P}_{a}+\frac{i}{2} \Omega_{. . \mu}^{a b} \mathbf{M}_{a b}$,

where $\mathbf{M}_{a b}$ are related with the six generators of the group $S O(3,1)$ with $\mathbf{J}_{a}=\epsilon_{a b c} \mathbf{M}_{b c}, \mathbf{K}_{a}=-\mathbf{M}_{o a}$ and $\mathbf{P}_{a}$ are the generators of the translational group $T(3,1)$. Here $\omega_{. \mu}^{a}$ and $\Omega_{. . \mu}^{a b}$ are connection 1 -forms of the $T(3,1)$ and $S O(3,1)$ group, respectively. The total field strength tensor can be defined as

$F_{\mu \nu}=\left[D_{\mu}, D_{\nu}\right]=T_{. \mu \nu}^{a} P_{a}+\frac{1}{2} R_{. . \mu \nu}^{a b} M_{a b}$,

with the torsion tensor

$T_{. \nu \mu}^{a}=\partial_{\nu} \omega_{. \mu}^{a}-\partial_{\mu} \omega_{. \nu}^{a}+\left(\Omega_{. e v}^{a} \omega_{. \mu}^{e}-\Omega_{. e \mu}^{a} \omega_{. \nu}^{e}\right)$,

and the curvature tensor

$R_{. b \nu \mu}^{a}=\partial_{\nu} \Omega_{. b \mu}^{a}-\partial_{\mu} \Omega_{. b v}^{a}+\left(\Omega_{. e v}^{a} \Omega_{. b \mu}^{e}-\Omega_{. e \mu}^{a} \Omega_{. b v}^{e}\right)$.

Through contraction with tetrads, tensors can be transformed to spacetime indexed forms as e.g. $v^{\mu}=e_{a}^{\mu} v^{a}, R_{. \lambda v \mu}^{\varrho}=$ $e_{a}^{\varrho} e_{\lambda}^{b} R_{. b \nu \mu}^{a}$ and the lower frame index $v_{a}$ can be raised by the Lorentz metric $v^{a}=\eta^{a b} v_{b}$. The connections $\omega_{. \mu}^{a}$ and $\Omega_{. . \mu}^{a b}$ are fundamental structure functions characterizing the specific tangent bundle.

Parallel transport of a tangent vector $v^{a}$ from a point $x$ in the spacetime manifold to a neighboring point $x^{\prime}$ is defined by the covariant derivative

$D_{\mu} v^{a}=\partial_{\mu} v^{a}+\Omega_{. \mu b}^{a} v^{b}$,

where $\Omega_{. \mu b}^{a}$ is called a frame connection. On the other hand, the covariant derivative of vectors which refer to the coordinate basis can be written as

$D_{\mu} v^{v}=\partial_{\mu} v^{v}+\Gamma_{. \mu \rho}^{\nu} v^{\rho}$,

with the coordinate connection $\Gamma_{. \mu \rho}^{\nu}$. The coordinate connection $\Gamma_{. \mu \lambda}^{\rho}$ is connected with the frame connection $\Omega_{. b \mu}^{a}$ by requiring $D_{\mu} e_{\nu}^{a}=\partial_{\mu} e_{\nu}^{a}-\Gamma_{. \mu \nu}^{\rho} e_{\varrho}^{a}+\Omega_{. \mu b}^{a} e_{\nu}^{b}=0$ from which the following relation can be derived:

$\Omega_{. b \mu}^{a}=e_{\nu}^{a} \partial_{\mu} e_{b}^{\nu}+e_{\nu}^{a} \Gamma_{. \rho \mu}^{\nu} e_{b}^{\varrho}$.

For the description of gravity tetrads $e_{b}^{v}$ and a specific coordinate connection $\Gamma_{. \mu \lambda}^{\rho}$ has to be defined. For a manifold with vanishing metricity described by the condition $D_{\lambda} g_{\mu \nu}=$ $\partial_{\lambda} g_{\mu \nu}-\Gamma_{. \mu \lambda}^{\rho} g_{\varrho \nu}-\Gamma_{. \nu \lambda}^{\varrho} g_{\mu \rho}=0$ one gets [10]

$\Gamma_{. \mu \nu}^{\rho}=\widetilde{\Gamma}_{. \mu \nu}^{\varrho}+K_{. \mu \nu}^{\varrho}$,

where $\widetilde{\Gamma}_{. \mu \nu}^{\rho}$ is the Levi-Civita connection and $K_{. \mu \nu}^{\varrho}$ is the contortion tensor,

$K_{. \mu \nu}^{\varrho}=\frac{1}{2}\left(T_{\nu . \mu}^{\cdot e}+T_{\mu . \nu}^{\cdot \varrho}-T_{. \mu \nu}^{\varrho}\right)$,

with the torsion tensor $T_{. \mu \nu}^{\varrho}=\Gamma_{. \mu \nu}^{\varrho}-\Gamma_{. \nu \mu}^{\varrho}$. The underlying geometric structure with respect to translational and rotational transformations (6) of the tangent vectors leads to 
a Riemann-Cartan spacetime endowed with frame connections $\omega_{. v}^{a}$ and $\Omega_{. \mu b}^{a}$, nonvanishing curvature $R_{. b v \mu}^{a}$, nonvanishing torsion $T_{. v \mu}^{a}$ but vanishing metricity. The special case of a Riemann spacetime and the General Theory of Relativity (GTR) can be obtained from the above formulas by setting the torsion tensor to be identically vanishing. The coordinate connection $\Gamma_{. \rho \mu}^{v}$ then is given by the Levi-Civita connection $\widetilde{\Gamma}_{. \mu \nu}^{\varrho}$, related with the metric tensor $g_{\mu \nu}$. The frame connection $\Omega_{. \mu b}^{a}$ in this case is usually called a spin connection $\widetilde{\Omega}_{. b v}^{a}$; it is related with the Levi-Civita connection $\widetilde{\Gamma}_{. \mu \nu}^{\varrho}$ by Eq. (16). On the other hand, based on the symmetry with respect of translational transformations of tangent vectors a gauge theory of gravity has been developed by analogy with the internal symmetries [11-15]. This gauge gravity theory corresponds to the teleparallel gravity theory, which is an alternative but equivalent formulation of the GTR describing the very same gravitational field. In teleparallel gravity, the torsion is nonvanishing, acting as a gravitational force while the curvature $R_{. b v \mu}^{a}$ vanishes identically. The translational connections $\omega_{\mu}^{a}$ and the tetrad coframe $e_{\mu}^{a}$ turn out to be conceptional distinct entities, since it does not transform inhomogeneously with a gradient term under a gauge transformation [18]. The coframes depend on the translational connection in the form $e_{\mu}^{a}=\omega_{\mu}^{a}+D_{\mu} \xi^{a}, D_{\mu}=\partial_{\mu} \delta_{a b}+\Omega^{a}{ }_{\mu b}$ where $\xi^{a}=\xi^{a}(x)$ is a coset vector. Locally at a given point $x$ on the spacetime manifold one can transform $D_{\mu} \xi^{a}$ by a gauge tansformation into $\delta_{a \mu}$ (where $\delta$ is the Kronecker symbol). Note that the coframe $e_{\mu}^{a}$ induces a metric as an independent dynamically quantity. In the geometry of teleparallel gravity the coordinate connection $\Gamma_{. \mu \lambda}^{\rho}$ takes the form of the Weizenböck connection $\Gamma_{. \mu \lambda}^{\| \rho}={ }^{W} \Gamma_{. \mu \nu}^{\rho}(x)$, defined as

${ }^{W} \Gamma_{. \mu \nu}^{\rho}(x)=e_{a}^{\varrho}(x) \partial_{\nu} e_{\mu}^{a}(x)$.

If we consider only gravitational effects with the choice of the Weizenböck connection in teleparallel gravity a vector is parallel transported if its projection on the tetrads is proportional, regardless of the path connecting the two tangent spaces. This can we see from (15) with the substitution of $\Gamma_{. \mu \nu}^{\rho}(x)$ by ${ }^{W} \Gamma_{. \mu \nu}^{\rho}(x)$, which yields for the covariant derivative $D_{\mu}^{\|} v^{v}=e_{a}^{v}(x) \partial_{\mu} v^{a}$. In teleparallel gravity the connection coefficients $\Omega_{. b v}^{\| a}$ represents a pure inertial effect [14] where $\Omega_{. b v}^{\prime a}$ is the spin connection in teleparallel gravity. There exist a class of inertial frames in which $\Omega_{. b v}^{\| a}$ vanishes: $\Omega_{b v}^{\| a}=0$.

The Weizenböck torsion can be used to build up the Lagrangian of teleparallel gravity with quadratic Weizenböck scalars $[11,14,15]$, given by

$$
L=-\frac{h}{16 \pi}\left[\frac{1}{4} T^{a b c} T_{a b c}+\frac{1}{2} T^{a b c} T_{b a c}-T^{a} T_{a}\right],
$$

where $h=\operatorname{det}\left(e_{\mu}^{a}\right)$ and $T_{b}=T_{a b}^{a}$. The Lagrangian is up to a divergence equivalent to the Lagrangian of Einstein's GTR, this means the two theories are simply alternative formulations for the description of gravity but are based on different principles.

A principal bundle $P(M ; G)$ encodes the essential data of gauge transformations and the frame connection $\Omega_{. b v}^{a}$ and the $\omega_{. \mu}^{a}$ are additional structure functions that are attached to it and are in general independent defined on the existence of a metric ([8,9]). If the structure group $G=S O(3,1) \rtimes T(3,1)$ is restricted to the translational subgroup $T(3,1)$ Eqs. (12) and (13) can be taken with $\Omega_{. b \mu}^{a}$ everywhere put equal to zero. The torsion tensor is now determined by $T_{. v \mu}^{a}=$ $\partial_{\nu} \omega_{. \mu}^{a}-\partial_{\mu} \omega_{. v}^{a}$, which can be obtained by a specific gauge fixing and describes gravitation in the so-called pure teleparallel gravity theory. Note that the structure group of the tangent bundle is the larger group $G=S O(3,1) \rtimes T(3,1)$. This raises the question of the physical meaning of the other subgroup $S O(3,1)$. If the $\Omega_{. b \mu}^{a}$ do not vanish we find from (13) a non-Abelian field strength tensor which in teleparallel gauge gravity theory (based on the translational symmetry) is not related to gravity. In this paper the main hypothesis is elaborated that non-Abelian fields in electroweak interaction can be identified with the connection coefficients $\Omega^{a}{ }_{b \mu}$ arising from transformations along the tangent fiber axis described by the group $S O(3,1)$. This interpretation differs from Poincaré gravity gauge theory based on the localization of the Poincaré group as gauge group [16] (for a review see [17]). In this theory both the translational part and the rotational part of the local Poincaré group is related with gravity leading to a hypothetical generalized gravity theory, denoted as the Einstein-Cartan gravity theory. In the following we use the terms connection coefficients and gauge potentials in parallel as well as gauge transformations and tangent vector transformations.

\section{The generators on the little groups}

From now on we neglect gravity arising from the translational part $a^{a}(x)$ of the transformation of tangent vectors in (6). Since the action of $S O(3,1)$ on a tangent vector is not transitive, the vector space decomposes into different orbits with the little groups $S O(3), E(2)$ and $S O(1,2)$. The unitary representations $T_{L}(g)$ of the little groups $S O(3)$ and $E(2)$ are well known. The composition law of these so-called vector representations $T_{L}(g)$ satisfy the functional equation $T_{L}\left(g_{1}\right) T_{L}\left(g_{2}\right)=T_{L}\left(g_{1} g_{2}\right)$ and encode the law of group transformations on the set of vector states. However, it is well known that this composite law is too restrictive and leads in special cases to certain pathologies, as e.g. the Dirac equation is not invariant under the Poincaré group, but under its universal covering group. In quantum theory the physical 
symmetry of a group of transformations on a set of vector states has to preserve the transition probability between two vector states $\left|\prec \Phi, T_{L}(g) \Psi \succ\right|^{2}=|\prec \Phi, \Psi \succ|^{2}$. Therefore, as shown by Wigner [19] and systematically studied by Bargman [20], the problem of pathologies can be solved if the composite law given above is replaced by a weaker one: $T_{L}\left(g_{1}\right) T_{L}\left(g_{2}\right)=\varepsilon\left(g_{1}, g_{2}\right) T_{L}\left(g_{1} g_{2}\right)$ where $\varepsilon\left(g_{1}, g_{2}\right)$ is a complex-valued antisymmetric function of the group elements with $\left|\varepsilon\left(g_{1}, g_{2}\right)\right|=1$. Such representations are called projective representations. For the case of simply connected groups like the rotation group $S O(3)$ projective representations are obtained by replacing the group $S O(3)$ by its universal cover $S U$ (2). However, in the case of the non-semisimple Euclidean group $E(2)$ the covering group is not enough, one has to substitute this group by a larger group: the universal central extension $E^{c}(2)$, which includes in addition to the group elements of $E(2)$ the group $U(1)$ of phases factors $\varepsilon\left(g_{1}, g_{2}\right)$ with $\left|\varepsilon\left(g_{1}, g_{2}\right)\right|=1$. In general a central extension $G^{c}$ of a group $G$ with elements $(g, \varsigma) \in G^{c}$ and $g \in G, \varsigma \in U(1)$ satisfies the group law [20]

$$
\begin{aligned}
(g, \varsigma) & =\left(g_{1}, \varsigma_{1}\right) *\left(g_{2}, \varsigma_{2}\right) \\
& =\left(\left(g_{1} * g_{2}, \varsigma_{1} \varsigma_{2} \exp \left[i \xi\left(g_{1}, g_{2}\right)\right],\right.\right.
\end{aligned}
$$

where $\xi\left(g_{1}, g_{2}\right)$ is the two-cocycle satisfying the relation $\xi\left(g_{1}, g_{2}\right)+\xi\left(g_{1} * g_{2}, g_{3}\right)=\xi\left(g_{1}, g_{2} * g_{3}\right)+\xi\left(g_{2}, g_{3}\right)$, $\xi(e, g)=\xi(g, e)=0$.

The two-dimensional Euclidian group $E(2)=T(2) \otimes$ $S O(2)$ with $E(2)=\{(\alpha, \mathbf{a}) \mid \alpha \in R,(\bmod 2 \pi), \mathbf{a}=$ $\left.\left(a^{1}, a^{2}\right)^{T} \in R^{2}\right\}$ is a semi-direct product of translations and rotations of the two-dimensional Euclidian plane. Since the invariant subgroup $S O(2)$ is abelian, this group is not semisimple and not compact and the unitary representations are infinite dimensional. The representations of the group $E(2)$ constructed on the space of functions are well known. The action of the group $E(2)$ on a vector $\mathbf{Z}=\left(\xi_{1}, \xi_{1}\right)$ is given by

$$
\begin{aligned}
(\alpha, \mathbf{a})\left(\xi_{1}, \xi_{2}\right)= & \left(\xi_{1} \cos \alpha-\xi_{2} \sin \alpha+a^{1},\right. \\
& \left.\xi_{1} \sin \alpha+\xi_{2} \cos \alpha+a^{2}\right) .
\end{aligned}
$$

The most general (projective) representations of $E(2)$ cannot be obtained from its universal covering group but by the central extended group $E^{c}(2)$. This group has been studied previously as e.g. in [20-22]. The central extension embodies in addition a $\mathrm{U}(1)$ subgroup characterized by a complex parameter $\zeta=\exp (i \omega) . E^{c}(2)$ consists of elements $(\alpha, \mathbf{a}, \omega)$ with $(\alpha, \mathbf{a}) \in E(2), \omega \in R$. The action of the group $E^{c}(2)$ on a vector $\mathbf{Z}=\left(\xi_{1}, \xi_{2}, \beta\right)$ is described by [20-22]

$$
\begin{aligned}
(\alpha, \mathbf{a}, \omega)\left(\xi_{1}, \xi_{2}, \beta\right)= & \left(\xi_{1} \cos \alpha-\xi_{2} \sin \alpha+a^{1},\right. \\
& \xi_{1} \sin \alpha+\xi_{2} \cos \alpha+a^{2}, \\
& \left.\beta+\omega+\frac{1}{2} m\left(\alpha, \mathbf{a}, \xi_{1}, \xi_{2}\right)\right),
\end{aligned}
$$

where $m\left(\alpha, \mathbf{a}, \xi_{1}, \xi_{2}\right)$ is the two-cocycle which gives the desired central extension parametrized as

$$
\begin{aligned}
m\left(\alpha, \mathbf{a}, \xi_{1}, \xi_{2}\right)= & \left(a_{1} \xi_{1}+a_{2} \xi_{2}\right) \sin \alpha \\
& -\left(a_{1} \xi_{2}-a_{2} \xi_{1}\right) \cos \alpha .
\end{aligned}
$$

By using (23) and (24) we can find the infinitesimal transformations and the generators of the Lie algebra, which satisfy the following communication rules:

$\left[\mathbf{T}^{1}, \mathbf{T}^{2}\right]=i \mathbf{E}$,

$\left[\mathbf{T}^{1}, \mathbf{T}^{3}\right]=-i \mathbf{T}^{2}$,

$\left[\mathbf{T}^{2}, \mathbf{T}^{3}\right]=i \mathbf{T}^{1}$,

$\left[\mathbf{T}^{a}, \mathbf{E}\right]=0$.

Of particular interest are the generators on this group:

$\mathbf{T}^{1}=-i\left(\frac{\partial}{\partial \xi_{1}}+\frac{1}{2} \xi_{2} \frac{\partial}{\partial \beta}\right), \mathbf{T}^{2}=-i\left(\frac{\partial}{\partial \xi_{2}}-\xi_{1} \frac{1}{2} \frac{\partial}{\partial \beta}\right)$,

$\mathbf{T}^{3}=-i\left(\xi_{1} \frac{\partial}{\partial \xi_{2}}-\xi_{2} \frac{\partial}{\partial \xi_{1}}\right), \mathbf{E}=-i \frac{\partial}{\partial \beta}$.

The group Laplacian (Casimir operator) is determined by

$\boldsymbol{\Delta}=\left(\mathbf{T}^{1}\right)^{2}+\left(\mathbf{T}^{2}\right)^{2}+2 \mathbf{T}^{3} \mathbf{E}$.

Accordingly, the operator $\mathbf{E}$ is the center of the group. To derive a canonical basis we use the eigenfunction of $\mathbf{E}, \mathbf{T}^{3}$ and the Laplace operator $\Delta$ of $E^{c}(2)$. Using polar coordinates $\xi_{1}=\xi \cos \phi, \xi_{2}=\xi \sin \phi$ and $h_{n m \varkappa}(\xi, \beta, \phi)=$ $\exp (i \varkappa \beta)\left(\exp (i m \phi) g_{n m \varkappa}(\xi)\right.$ we find with the Laplacian (27) the following equation for $g_{n m \varkappa}(\xi)$ :

$$
\begin{aligned}
& {\left[\left(-\frac{1}{\xi} \frac{\partial}{\partial \xi} \xi \frac{\partial}{\partial \xi}+\frac{1}{\xi^{2}} m^{2}\right)+\varkappa^{2} \xi^{2}-2 \varkappa m\right] g_{n m \varkappa}(\xi)} \\
& =\epsilon_{n m \varkappa} g_{n m \varkappa}(\xi) .
\end{aligned}
$$

With the solutions of (28) we find for $h_{n m \varkappa}(\xi, \beta, \phi)$

$$
\begin{aligned}
h_{n m \varkappa}(\xi, \beta, \phi)= & N_{n m \varkappa}|\varkappa|^{|m| / 2} \exp (i \varkappa \beta)(\exp (i m \phi) \\
& \times \exp \left(-\frac{|\varkappa| \xi^{2}}{2}\right) \xi^{|m|} L_{n}^{|m|}\left(|\varkappa| \xi^{2}\right),
\end{aligned}
$$

with $N_{n m \varkappa}=\sqrt{\frac{\varkappa}{\pi}}\left(\frac{n !}{(|m|+n) !}\right)^{\frac{1}{2}}, \epsilon_{n m \varkappa}=4 \varkappa\left(n+\frac{1}{2}+\frac{1}{2}(m+\right.$ $|m|)$, with $n=0,1,2, \ldots, m=0, \pm 1, \pm 2, \ldots, \varkappa=$ $, \pm 1, \pm 2, \ldots$ Here $L_{n}^{|m|}(x)$ are the associated Legendre polynomials. The solutions $h_{n m \varkappa}$ form an ortho-normalized set and have a form analogous to the solutions of the 2D Schrödinger equation in the symmetric gauge for electrons in a constant magnetic field [23]. The group $E^{c}(2)$ is isomorphic to the quantum harmonic oscillator group studied e.g. in [24].

The special eigenvalue $\varkappa=0$ plays a particular role. The solution of (28) for $\varkappa=0$ is given by

$h_{m 00}^{\rho}=\frac{1}{\sqrt{2 \pi}} J_{|m|}(\rho \xi) \exp (i m \phi)$, 
and it agrees with the results for the group $E(2) . J_{|m|}(x)$ are the Bessel functions. The eigensolutions fall into two classes: the continuous series with $\rho^{2} \neq 0$ and the discrete series with $\rho^{2}=0$ and

$h_{0 m 0}^{0}(\phi)=\frac{1}{\sqrt{2 \pi}} \exp (i m \phi)$.

As described later the generator $\mathbf{T}_{3}$ corresponds to the hypercharge generator in the SM. It is convenient to introduce for $\mathbf{T}_{1}$ and $\mathbf{T}_{2}$ the generators $\mathbf{T}^{ \pm}=\frac{1}{\sqrt{2}}\left(\mathbf{T}^{1} \pm i \mathbf{T}^{2}\right)$. The action of the operator $\mathbf{T}^{+}$on the eigenfunctions $g_{n m \varkappa}$ increases the hypercharge from $m$ to $m+1$, but it simultaneously generates states with different family numbers $n$ : $\mathbf{T}^{+} g_{n m \varkappa}=$ $\sum_{i=0}^{n} A_{i} g_{i, m+1, \varkappa}$. Correspondingly the generator $\mathbf{T}^{-}$reduce the hypercharge: $\mathbf{T}^{-} g_{n m \varkappa}=\sum_{i=0}^{n} B_{i} g_{i, m-1, \varkappa} . A_{i}$ and $B_{i}$ are coefficients, respectively. The action of the operator product $\left(\mathbf{T}_{-} \mathbf{T}_{+}+\mathbf{T}_{+} \mathbf{T}_{-}\right)$on the eigenfunctions is given by

$\left(\mathbf{T}_{-} \mathbf{T}_{+}+\mathbf{T}_{+} \mathbf{T}_{-}\right) g_{n m \varkappa}(\xi, \phi, \beta)=q_{B} g_{n m \varkappa}(\xi, \phi, \beta)$,

with $q_{B}(n, m, \varkappa)=4 \varkappa\left[n+\frac{1}{2}(1+|m|)\right]$.

The generators $\mathbf{J}_{1}, \mathbf{J}_{2}, \mathbf{J}_{3}$ on the group $S U(2)$ are well known and, by using the Laplacian $\Delta=\left(\mathbf{J}_{1}^{2}+\mathbf{J}_{2}^{2}+\mathbf{J}_{3}^{2}\right)$ on this group, all finite-dimensional representations can be found (see e.g. [25,26]. The application of the operators $\mathbf{J}_{ \pm}=$ $2^{-1 / 2}\left(\mathbf{J}^{1} \pm i \mathbf{J}^{2}\right)$ and $\mathbf{J}_{3}$ on the eigenfunctions of the Laplacian $\Delta$ leads to

$$
\begin{aligned}
\mathbf{J}_{ \pm} f_{. j_{3}}^{j} & =\frac{1}{2}\left[j(j+1)-j_{3}\left(j_{3} \pm 1\right]^{1 / 2} f_{j_{3} \pm 1}^{j},\right. \\
\mathbf{J}_{3} f_{j_{3}}^{j} & =j^{3} f_{j_{3}}^{j},
\end{aligned}
$$

where $j=\left(-j_{3} \cdot-j_{3}+1, \ldots, j_{3}\right)$ are the isospin quantum numbers, $j_{3}$ is the projection on the third isospin axis. Moreover, we find

$\left(\mathbf{J}_{+} \mathbf{J}_{-}+\mathbf{J}_{-} \mathbf{J}_{+}\right) f_{. j_{3}}^{j}=q_{W} f_{. j_{3}}^{j}$,

with $q_{W}=\left[j(j+1)-j_{3}^{2}\right]$. Using the parametrization $z_{1}=$ $\cos \frac{\theta}{2} \exp [i(\psi-\varphi) / 2], z_{2}=i \sin \frac{\theta}{2} \exp [i(\psi+\varphi)]$ we find for $j=1 / 2, j_{3}=1 / 2$ from the $\mathrm{SU}(2)$ Laplacian $f_{j_{3}}^{j}=z_{1}$ and for $j=1 / 2, j_{3}=-1 / 2: f_{j_{3}}^{j}=z_{2}$. For the isospin numbers $j=1, j_{3}=1$ we have $f_{1}^{1}=\left(z_{1}\right)^{2} / \sqrt{2}$, for $j_{3}=0$ one finds $f_{1}^{0}=z_{1} z_{2}$, and $j_{3}=-1$ yields $f_{-1}^{1}=\left(z_{2}\right)^{2} / \sqrt{2}$. The eigenfunctions for the general case for the isospin $j$ are given by [26]

$f_{j j_{3}}=\left(\frac{1}{\left(j+j_{3}\right) !\left(j-j_{3}\right) !}\right)^{1 / 2} z_{1}^{j+j_{3}} z_{2}^{j-j_{3}}$.

In traditional quantum field theory the internal degrees of freedom, such as isospin, hypercharge or color, are described by spacetime depending multi-component fields taking into account the vertical structure given by the internal gauge groups by the corresponding Lie-algebra representation. In the TB description, different from the two-component formalism, the basic objects on the group $S U$ (2) (which here is interpreted as the isospin group) are functions $\Phi\left(x, z_{1}, z_{2}\right)$ depending both on the coordinate $x$ of the spacetime manifold and the complex coordinates $z_{1}=z_{1}(\theta, \varphi, \psi), z_{2}=$ $z_{2}(\theta, \varphi, \psi)$. General functions on the group manifold can be decomposed into the form

$\Phi\left(x, z_{1}, z_{2}\right)=\sum_{j_{3}=0}^{2 j} \phi_{j_{3}}(x) f_{j_{3}}^{j}\left(z_{1}, z_{2}\right)$.

The standard description for $j_{3}= \pm \frac{1}{2}$ by two-component functions $\left(\phi_{1}(x), \phi_{2}(x)\right)^{T}$ is obtained from (37) by the projection into the configuration space by using the relation

$\Phi\left(x, z_{1}, z_{2}\right)=\left(z_{1}, z_{2}\right)\left(\begin{array}{l}\phi_{1}(x) \\ \phi_{2}(x)\end{array}\right)$.

For finite-dimensional representations of the group $S U$ (2) the above presented description by using coordinates on the group manifold $z^{1}$ and $z^{2}$ is equivalent to the multicomponent description in nonabelian field theory. However, for a noncompact group as the group $E^{c}(2)$ a multicomponent representation is not favorable and the tangent bundle approach using coordinates of the tangent space is more convenient.

\section{Lagrangians on the tangent bundle}

\subsection{Lagrangian of Gauge fields on the group} $S U(2) \otimes E^{c}(2)$

Based on the above described orbit decomposition and using the group $G=S U(2) \otimes E^{c}(2)$ the covariant derivative (10) with $\omega_{\mu}^{a}=0$ can be rewritten as

$$
\begin{aligned}
D_{\mu}= & \frac{\partial}{\partial x^{\mu}}+i g_{1} A_{\mu}^{a}(x, u) \mathbf{J}_{a}+i g_{2} \frac{1}{2} B_{\mu}^{a}(x, u) \mathbf{T}_{a} \\
& +i g_{3} C_{\mu}(x, u) \mathbf{E},
\end{aligned}
$$

where $\mathbf{J}_{a}$ are the generators of the group $S U(2)$ and $\mathbf{T}_{a}$ and $\mathbf{E}$ are the operators (26) on the group $E^{c}(2) . A_{\mu}^{a}(x, u), B_{\mu}^{a}(x, u)$ and $C_{\mu}(x, u)$ are the frame connection coefficients (gauge potentials). The gauge field strength tensors can be obtained from the commutators $\left[\mathbf{D}_{\mu}, \mathbf{D}_{v}\right]$. Let us first consider the gauge fields for the group $E^{c}(2)$. The following relations for the field strength tensors $B_{\mu \nu}^{a}(x, u)$ and $C_{\mu \nu}(x, u)$ of the generators $\mathbf{T}_{a}$ and $\mathbf{E}$ can be derived:

$$
\begin{aligned}
B_{\mu \nu}^{ \pm}= & \frac{\partial}{\partial x^{\mu}} B_{v}^{ \pm}-\frac{\partial}{\partial x^{\nu}} B_{\mu}^{ \pm} \\
& \pm g_{2} i\left(B_{\mu}^{ \pm} B_{v}^{3}-B_{\mu}^{3} B_{v}^{ \pm}\right), \\
B_{\mu \nu}^{3}= & \frac{\partial}{\partial x^{\mu}} B_{v}^{3}-\frac{\partial}{\partial x^{\nu}} B_{\mu}^{3}
\end{aligned}
$$


$C_{\mu \nu}=\frac{\partial}{\partial x^{\mu}} C_{\nu}-\frac{\partial}{\partial x^{\nu}} C_{\mu}-g_{2} i\left(B_{\mu}^{+} B_{v}^{-}-B_{\mu}^{-} B_{v}^{+}\right)$,

where $B_{\mu}^{ \pm}=2^{-1 / 2}\left(B_{\mu}^{1} \pm i B_{\mu}^{2}\right)$.

Recently the non-semisimple group $E^{c}(2)$ was found to be relevant in $1+1$ gravity theory [27], for the construction of string background in the Wess-Zumino-Witten (WZW) model, in 3D Chern-Simon theory [28-30], and in YangMills theory [29]. Gauge theory for a group with generators $\mathbf{L}_{a}$ and commutators $\left[\mathbf{L}_{a}, \mathbf{L}_{b}\right]=i f_{a b}^{c} \mathbf{L}_{c}$ requires a bilinear form $g_{a b}$ which is symmetric, invariant with respect of gauge transformations, and non-degenerate so that there exists an inverse matrix. For semisimple groups the invariant bilinear form is given by the Killing form and it is proportional to the Kronecker symbol $g_{a b}^{K}=f_{a c}^{d} f_{b d}^{c}=\delta_{a b}$. For the nonsemisimple group $E^{c}(2)$ the Killing form is degenerate and it is given by $g_{a b}^{K}=\delta_{a 3}$. Nevertheless there exists another form of an invariant product for this gauge group. Let us determine the general conditions for a non-degenerate invariant product.

The Lagrangian of the gauge fields must be a quadratic combination of the field strength tensor $B_{\mu \nu}^{a}$. Lorentz covariance restricts its form to

$L_{g}=-\frac{1}{4} g_{a b} B_{\mu \nu}^{a} B^{b \mu \nu}$,

with the invariant symmetric metric $g_{a b}\left(g_{a b}=g_{b a}\right)$. Under an infinitesimal gauge transformation the field strengths transform like $\delta B_{\mu \nu}^{a}=-f_{b c}^{a} B_{\mu \nu}^{b} \theta^{c}$. The Lagrangian must be gauge-invariant, therefore the following condition has to be fulfilled:

$g_{a b} B_{\mu \nu}^{a} f_{d e}^{b} B^{\mathrm{d} \mu \nu} \theta^{e}=0$.

Accordingly the metric $g_{a b}$ must satisfy the following condition:

$g_{a c} f_{b d}^{c}+g_{b c} f_{a d}^{c}=0$.

For the group $\mathrm{E}^{c}(2)$ with the commutation rule (25) for the generators $\mathbf{L}_{a}=\mathbf{T}_{a}(a=1,2,3)$ and $\mathbf{L}_{4}=\mathbf{E}$ the nonzero coefficients $f_{a b}^{c}$ are given by $f_{12}^{4}=-f_{21}^{4}=1, f_{23}^{1}=$ $-f_{32}^{1}=1, f_{13}^{2}=-f_{31}^{2}=-1$. Accordingly one can derive a non-degenerate symmetric invariant bilinear form $g_{a b}=g_{b a}$ given by [27-29]

$g_{a b}^{0}=\left[\begin{array}{cccc}1 & 0 & 0 & 0 \\ 0 & 1 & 0 & 0 \\ 0 & 0 & 0 & 1 \\ 0 & 0 & 1 & 0\end{array}\right]$.

The most general invariant quadratic form is a linear combination of the metric $g_{a b}^{0}$ and the Killing form $g_{a b}^{K}=\delta_{a 3}$ given by

$$
g_{a b}=\left[\begin{array}{cccc}
1 & 0 & 0 & 0 \\
0 & 1 & 0 & 0 \\
0 & 0 & k & 1 \\
0 & 0 & 1 & 0
\end{array}\right], g^{a b}=\left[\begin{array}{cccc}
1 & 0 & 0 & 0 \\
0 & 1 & 0 & 0 \\
0 & 0 & 0 & 1 \\
0 & 0 & 1 & -k
\end{array}\right],
$$

with an arbitrary parameter $k$. The metric $g_{a b}$ induces a family of non-degenerate invariant quadratic forms of Lorentzinvariant vectors $v_{\mu}^{a}$ :

$I=v_{\mu}^{1} v^{1 \mu}+v_{\mu}^{2} v^{2 \mu}+k v_{\mu}^{3} v^{3 \mu}+2 v_{\mu}^{3} v^{4 \mu}$.

With a scaling of $g_{a b} \rightarrow \frac{1}{k} \widetilde{g}_{a b}$ and $k \gg 1$ the invariant product is given by

$I_{0}=v_{\mu}^{3} v^{3 \mu}$.

The generator $\mathbf{T}^{3}$ of the group $E^{c}(2)$ corresponds to the hypercharge operator in the SM and the Lagrangian of the corresponding gauge field is described by the $U(1)$ group:

$L_{g}^{E^{c}}=-\frac{1}{4} B_{\mu \nu}^{3} B^{3 \mu \nu}$.

In the general case the invariant product can be diagonalized by the transformation $v_{\mu}^{3}=\frac{1}{\sqrt{2}}\left(\cos \alpha v_{\mu}^{\prime 3}-\sin \alpha v_{\mu}^{\prime 4}\right)$, $v_{\mu}^{4}=\frac{1}{\sqrt{2}}\left(\sin \alpha v_{\mu}^{\prime 3}+\cos \alpha v_{\mu}^{\prime 4}\right)$ with $\tan 2 \alpha=\frac{2}{k}$. Then the quadratic form is given by

$$
\begin{aligned}
I & =g_{a b} v_{\mu}^{a} v^{b \mu}, \\
& =v_{\mu}^{1} v^{1 \mu}+v_{\mu}^{2} v^{2 \mu}+a_{3} v_{\mu}^{\prime 3} v^{\prime 3 \mu}-a_{4} v_{\mu}^{\prime 4} v^{\prime 4 \mu},
\end{aligned}
$$

with $a_{3}=k \cos ^{2} \alpha+2 \cos \alpha \sin \alpha, a_{4}=-k \sin ^{2} \alpha+$ $2 \sin \alpha \cos \alpha$. With the choice $a_{3}=a_{4}$ we get $k=0, \alpha=\pi / 4$ and $a_{3}=a_{4}=1$.

Using (45) the gauge Lagrangian of the $E^{c}(2)$ model is given by

$$
\begin{aligned}
L_{E^{c}} & =-\frac{1}{4} g_{a b} B_{\mu \nu}^{a} B^{b \mu \nu} \\
& =-\frac{k}{4} B_{\mu \nu}^{3} B^{3 \mu \nu}-\frac{1}{2}\left(B_{\mu \nu}^{+} B^{-\mu \nu}+B_{\mu \nu}^{3} C^{\mu \nu}\right) .
\end{aligned}
$$

The Lagrangian is invariant under the gauge transformations

$\delta B_{\mu}^{ \pm}=\partial_{\mu} \theta^{ \pm} \mp g_{2} i\left(B_{\mu}^{3} \theta^{ \pm}-\theta^{3} B_{\mu}^{ \pm}\right)$,

$\delta B_{\mu}^{3}=\partial_{\mu} \theta^{3}$,

$\delta C_{\mu}=\partial_{\mu} \theta^{C}+g_{2} i\left(\theta^{+} B_{\mu}^{-}-\theta^{-} B_{\mu}^{+}\right)$,

where $\theta^{3}, \theta^{ \pm}, \theta^{C}$ are arbitrary spacetime depending functions.

The Lagrangian (50) is not positive definite and leads to negative terms in the Hamiltonian and to the occurrence of particles with un-physical negative norm. This situation is analogical to the case of gauge field quantization in covariant gauge of the $\mathrm{SM}$ with the gauge group $S U(2) \otimes U(1)$, where due to the form of the Lorentz metric the time-like component $B_{0}^{a}$ must correspond to negative metric particles. Quantization requires one to choose a specific gauge by adding terms like $\left(\partial_{\mu} B_{\mu}^{a}\right)^{2}$ and $\left(\partial_{\mu} C_{\mu}\right)^{2}$ to the Lagrangian for a covariant gauge which breaks the gauge invariance and introduce new un-physical fields. These so-called Fadeev-Popov ghost 
fields cancel the un-physical gauge field components with negative norm.

A diagonal form of the Lagrangian (50) can be achieved by the transformations

$C_{\mu}=\left(C_{\mu}^{+}-C_{\mu}^{-}\right)$

$B_{\mu}^{3}=\left(C_{\mu}^{+}+C_{\mu}^{-}\right)$

or the corresponding field strengths

$C_{\mu \nu}=\left(C_{\mu \nu}^{+}-C_{\mu \nu}^{-}\right)$,

$B_{\mu \nu}^{3}=\left(C_{\mu \nu}^{+}+C_{\mu \nu}^{-}\right)$.

The Lagrangian of gauge particles with a gauge fixing term and the Fadeev-Popov ghosts is for $k=0$ given by

$$
\begin{aligned}
L_{E^{c}}=- & \frac{1}{4}\left\{2 B_{\mu \nu}^{+} B^{-\mu \nu}+C_{\mu \nu}^{+} C^{+\mu \nu}-C_{\mu \nu}^{-} C^{-\mu \nu}\right. \\
& +\frac{1}{2 \xi}\left(\partial^{\mu} B_{\mu}^{+}\right)^{2}+\frac{1}{2 \xi}\left(\partial^{\mu} B_{\mu}^{-}\right)^{2}+\frac{1}{2 \zeta}\left(\partial^{\mu} C_{\mu}^{+}\right)^{2} \\
& +\frac{1}{2 \zeta}\left(\partial^{\mu} C_{\mu}^{-}\right)^{2}-\left(\partial_{\mu} \bar{c}^{3}\right) c^{3} \\
& -\partial_{\mu} \bar{c}^{+} c^{-}-\left(\partial_{\mu} \bar{c}^{-}\right) c^{+} \\
& -\left(\partial_{\mu} \bar{c}^{4}\right) c^{4}+i g_{2}\left(\partial_{\mu} \bar{c}^{4}\right)\left(c^{+} B^{-\mu}-c^{-} B^{+\mu}\right) \\
& -i g_{2}\left[\left(\partial_{\mu} \bar{c}^{+}\right) c^{-}-\left(\partial_{\mu} \bar{c}^{-}\right) c^{+}\right]\left(C^{+\mu}+C^{-\mu}\right) \\
& \left.-i g_{2}\left[\left(\partial_{\mu} \bar{c}^{+}\right) B^{-\mu}-\left(\partial_{\mu} \bar{c}^{-}\right) B^{+\mu}\right] c^{3}\right\},
\end{aligned}
$$

where $c^{ \pm}=\frac{1}{\sqrt{2}}\left(c^{1} \pm i c^{2}\right), c_{3}, c_{4}$ and $\bar{c}^{ \pm}, \bar{c}^{3}, \bar{c}^{4}$ are the anticommuting ghost fields and $\zeta$ is the gauge parameter. The cancellation of the un-physical gauge particles with negative norm by the Fadeev-Popov ghost particles can be proven by the BRST symmetry in a gauge-invariant form. Quantization of non-semisimple gauge groups has been studied in [29,3133]. In [29] one-loop radiative corrections for the Yang-Mills model with the $E^{c}(2)$ gauge group were computed. It was shown that there is no two- and higher-loop re-normalization and the full quantum effective action is given by the one-loop term with the divergent part that can be eliminated by a field redefinition.

The field strength tensor for the group $S U(2)$ is given by

$W_{\mu \nu}^{ \pm}=\frac{\partial}{\partial x^{\mu}} W_{\nu}^{ \pm}-\frac{\partial}{\partial x^{\nu}} W_{\mu}^{ \pm}$ $\pm g_{1} i\left(W_{\mu}^{ \pm} B_{v}^{3}-B_{\mu}^{3} W_{v}^{ \pm}\right)$,

$B_{\mu \nu}^{3}=\frac{\partial}{\partial x^{\mu}} B_{\nu}^{3}-\frac{\partial}{\partial x^{\nu}} B_{\mu}^{3}-g_{1} i\left(W_{\mu}^{+} W_{\nu}^{-}-W_{\mu}^{-} W_{\nu}^{+}\right)$.

The total gauge Lagrangian on the TB is $L_{g}=L_{E^{c}}+$ $L_{S U(2)}$ where the Lagrangian of the group $S U(2)$ with the inclusion of ghost fields is given by

$L_{S U(2)}=-\frac{1}{4}\left\{2 W_{\mu \nu}^{+} W^{-\mu \nu}+A_{\mu \nu}^{3} A^{3 \mu \nu}+\frac{1}{2 \xi}\left(\partial^{\mu} W_{\mu}^{+}\right)^{2}\right.$

$$
\begin{aligned}
& +\frac{1}{2 \xi}\left(\partial^{\mu} W_{\mu}^{-}\right)^{2}+\frac{1}{2 \zeta}\left(\partial^{\mu} A_{\mu}^{3}\right)^{2} \\
& -\left(\partial_{\mu} \bar{\omega}^{3}\right) \omega^{3}-\partial_{\mu} \bar{\omega}^{+} \omega^{-}-\left(\partial_{\mu} \bar{\omega}^{-}\right) \omega^{+} \\
& +i g_{2}\left(\partial_{\mu} \bar{\omega}^{3}\right)\left(\omega^{+} W^{-\mu}-\omega^{-} W^{+\mu}\right) \\
& -i g_{2}\left[\left(\partial_{\mu} \bar{\omega}^{+}\right) \omega^{-}-\left(\partial_{\mu} \bar{\omega}^{-}\right) \omega^{+}\right] B^{3 \mu} \\
& \left.-i g_{2}\left[\left(\partial_{\mu} \bar{\omega}^{+}\right) W^{-\mu}-\left(\partial_{\mu} \bar{\omega}^{-}\right) W^{+\mu}\right] \omega^{3}\right\},
\end{aligned}
$$

with the field strength tensors of the $S U$ (2) gauge field $W_{\mu}^{ \pm}=$ $2^{-1 / 2}\left(A_{\mu}^{1} \pm i A_{\mu}^{2}\right)$ and $A_{\mu}^{3} \cdot \bar{\omega}^{a}$ and $\omega^{a}$ are a set of independent anticommuting variables of the ghosts.

5.2 Lagrangians of matter fields on the group $S U(2) \otimes E^{c}(2)$

In the Lagrangian of the Higgs scalar particles $L_{H}=$ $\left(D_{\mu} \Phi_{H}\right)^{\dagger}\left(D^{\mu} \Phi_{H}\right)$ the electromagnetic field should not couple to the neutrino and should be diagonalized. Substituting (39) into the Lagrangian of the Higgs boson new mixing terms appear and the diagonalization requires that the fields $A_{\mu}^{3}, B_{\mu}^{3}$ and $C_{\mu}$ have to be transformed to new fields expressed by the relations

$B_{\mu}^{3}=\cos \theta_{W} A_{\mu}-\sin \theta_{W}\left(\cos \theta_{D} Z_{\mu}-\sin \theta_{D} E_{\mu}^{c}\right)$,

$A_{\mu}^{3}=\sin \theta_{W} A_{\mu}+\cos \theta_{W}\left(\cos \theta_{D} Z_{\mu}-\sin \theta_{D} E_{\mu}^{c}\right)$,

$C_{\mu}^{3}=\sin \theta_{D} Z_{\mu}+\cos \theta_{D} E_{\mu}^{c}$,

where $\theta_{W}$ is the Weinberg angle, $g_{2}=g \sin \theta_{W}, g_{1}=$ $g \cos \theta_{W}, g=\left(\left(g_{1}\right)^{2}+\left(g_{2}\right)^{2}\right)^{1 / 2}, e=g \cos \theta_{W} \sin \theta_{W}$ and $\theta_{D}$ is defined by $\tan 2 \theta_{D}=g g_{3} m_{H} \varkappa_{H} /\left[\left(g_{3} \varkappa_{H}\right)^{2}-\left(\frac{m_{H}}{2} g\right)^{2}\right]$, $m_{H}$ and $\varkappa_{H}$ are the IQNs of the Higgs particle. The covariant derivative (39) can be rewritten as

$$
\begin{aligned}
\mathbf{D}_{\mu}= & \frac{\partial}{\partial x^{\mu}}+i\left[W_{\mu}^{+} \mathbf{Q}_{W^{-}}+W_{\mu}^{-} \mathbf{Q}_{W^{+}}\right)+B_{\mu}^{+} \mathbf{Q}_{B^{-}} \\
& \left.+B_{\mu}^{-} \mathbf{Q}_{B^{+}}+Z_{\mu} \mathbf{Q}_{Z}+A_{\mu} \mathbf{Q}+E_{\mu}^{c} \mathbf{Q}_{E^{c}}\right],
\end{aligned}
$$

with $W_{\mu}^{ \pm}=2^{-1 / 2}\left(W_{\mu}^{1} \pm i W_{\mu}^{2}\right)$ and $B_{\mu}^{ \pm}=2^{-1 / 2}\left(B_{\mu}^{1} \pm i B_{\mu}^{2}\right)$. The following operators are introduced:

$$
\begin{aligned}
\mathbf{Q}= & \left(\mathbf{J}_{3}+\frac{1}{2} \mathbf{T}_{3}\right), \\
\mathbf{Q}_{Z}= & g \cos \theta_{D}\left(\cos ^{2} \theta_{W} \mathbf{J}_{3}-\sin ^{2} \theta_{W} \frac{1}{2} \mathbf{T}_{3}\right) \\
& +g_{3} \sin \theta_{D} \mathbf{E}, \\
\mathbf{Q}_{W^{ \pm}}= & g_{1} \mathbf{J}^{ \pm}, \mathbf{Q}_{B^{ \pm}}=g_{2} \frac{1}{2} \mathbf{T}^{ \pm}, \\
\mathbf{Q}_{E^{c}}= & g\left(-\cos ^{2} \theta_{W} \mathbf{J}_{3}+\sin ^{2} \theta_{W} \frac{1}{2} \mathbf{T}_{3}\right) \sin \theta_{D} \\
& +g_{3} \cos \theta_{D} \mathbf{E} .
\end{aligned}
$$

Any scalar function $\Phi(x, u)$ defined on the fiber bundle can be expanded into the form $\Phi=\sum_{M}\left(\phi_{M}(x) \chi_{M}(u)+\right.$ 
$\left.\phi_{M}^{\dagger}(x) \chi_{M}^{*}(u)\right)$ depending on the coordinates of the spacetime manifold $x$ and the eigenfunctions $\chi_{M}(u)=h_{l m \varkappa}(\xi, \phi, \beta)$ $f_{j j_{3}}\left(z_{1}, z_{2}\right)$ of the Laplacian of the group $S U(2) \otimes E^{c}(2)$ defined by (29) and (36). Besides we introduced the following symbol for the IQNs: $M=\left(n, m, \varkappa, j, j_{3}\right)$. The total Lagrangian can be presented by

$$
L=L_{l}+L_{H}+L_{g}+L_{\text {Yuk }} .
$$

Here the Lagrangian of the leptons $L_{l}$ is defined in the chiral representation as

$$
\begin{aligned}
L_{l}= & i \Psi_{s}^{l \dagger}(x, u) \sigma_{s}^{\mu} \\
& \times\left\{\frac{\partial}{\partial x^{\mu}}+i\left[W_{\mu}^{+} \mathbf{Q}_{W^{-}}+W_{\mu}^{-} \mathbf{Q}_{W^{+}}\right.\right. \\
& \left.\left.+Z_{\mu} \mathbf{Q}_{z}+A_{\mu} \mathbf{Q}+E_{\mu}^{c} \mathbf{Q}_{E^{c}}\right]\right\} \Psi_{s}^{l}(x, u),
\end{aligned}
$$

with the helicity $s=\{L, R\}$ and $\sigma_{R}^{\mu}=\left(\sigma^{0}, \sigma^{i}\right), \sigma_{L}^{\mu}=$ $\left(\sigma^{0},-\sigma^{i}\right)$ and $\Psi_{s}^{l}(x, u)=\sum_{M}\left(\psi_{M, s}^{-}(x) \chi_{M}(u)+\psi_{M, s}^{+}\right.$ $\left.(x) \chi_{M}^{*}(u)\right)$. Note that gauge fields and fermions or scalars can carry different IQNs. As shown below, the known SM gauge particles as the $Z$ and $W^{ \pm}$bosons and the $E^{c}$ gauge boson carry the IQN $\varkappa=0$, but due to the existence of families of SM leptons they carry nonzero $\mathrm{E}^{c}$-charges $\varkappa \neq 0$. The interaction of leptons with the $B^{ \pm}$bosons is forbidden because of a selection rule, as discussed below.

The Lagrangian of the SM Higgs particles $\Phi_{H}=$ $\Phi_{H}(x, u)$ (with $I_{3}=-1 / 2, m=1$ ) in the unitary gauge is given by

$$
\begin{aligned}
L_{H}= & \partial_{\mu} \Phi_{H}^{\dagger} \partial^{\mu} \Phi_{H}+\left|\Phi_{H}\right|^{2} \\
& \times\left[\frac{g_{1}^{2}}{2} W_{\mu}^{+} W^{\mu-}+g_{2}^{2} q_{B} B_{\mu}^{+} B^{\mu-}+E_{\mu}^{c} E^{c \mu}\left|Q_{E^{c}}\right|^{2}\right. \\
& \left.+\left|Q_{Z}\right|^{2} Z_{\mu} Z^{\mu}\right]+V\left(\Phi_{H}\right),
\end{aligned}
$$

with $Q_{Z}=-\frac{1}{2} \cos \theta_{D} g+g_{3} \varkappa_{H} \sin \theta_{D}, Q_{E^{c}}=g \sin \theta_{D} / 2+$ $g_{3} \varkappa_{H} \cos \theta_{D}, q_{B}=4 \varkappa_{H}(n+1)$. The interaction of the SM Higgs with the $W$ bosons remains the same as in the SM. The $E^{c}$ and $B^{ \pm}$gauge bosons are not decoupled from the SM particles; according to (62) there is a coupling of the new $E^{c}$ and $B_{\mu}^{ \pm}$bosons to the SM Higgs boson. In (62) the SM Higgs self-interaction potential $V\left(\Phi_{H}\right)=-\mu^{2}\left|\Phi_{H}\right|^{2}$ $+\lambda\left|\Phi_{H}\right|^{4}$ is included.

We denote the left- handed lepton family by $E_{\mathrm{L}}=$ $\left(e_{\mathrm{L}}, \mu_{\mathrm{L}}, \tau_{\mathrm{L}}\right)$ and the right-handed family by $E_{\mathrm{R}}=\left(e_{\mathrm{R}}\right.$, $\left.\mu_{\mathrm{R}}, \tau_{\mathrm{R}}\right)$. The SM Yukawa interaction $L_{\text {Yuk }}$ term is given by

$L_{\text {Yuk }}=-\Sigma_{n_{1} n_{2}} \sigma_{n_{1} n_{2}} \Psi_{M_{E_{L}}}^{\dagger} \Phi_{M_{H}} \Psi_{N_{E_{R}}}+$ h.c.

with $\sigma_{n_{1} n_{2}}$ as constant coupling coefficient and with the IQNs $M_{E_{L}}=\left\{n_{1}, m=-1, j=j_{3}=\frac{1}{2}, \varkappa_{E_{L}}\right\}, N_{E_{R}}=\left\{n_{2}, m=\right.$ $\left.-2, j=0, \varkappa_{E_{R}}\right\}$ and with the IQN of the Higgs particle: $M_{H}=\left\{n_{H}, m_{H}=1, j_{3}=-\frac{1}{2} \cdot \varkappa_{H}\right\}$. An assumption concerning the IQN $\varkappa$ of leptons and the Higgs particles will be discussed later.

The self-interaction term $V\left(\Phi_{S}\right)$ and $L_{\text {Yuk }}$ do not arise in the TB tree-level approximation but are included for phenomenological reasons in the same way as in the SM. The microscopic foundation of these phenomenological terms is an unsolved problem in the SM as well as in the approach here presented.

\section{Quantization on the tangent bundle}

On the tangent bundle one-particle states of the fermion Dirac field $\Psi_{f}(x, u)=\sum_{M, s}\left(\psi_{M, s}(x) \chi_{M}(u)+\psi_{M, s}^{\dagger}(x) \chi_{M}^{*}(u)\right)$ labeled by the three-momentum $\mathbf{p}$ are described by

$$
\begin{aligned}
\Psi_{f}(x, u)= & \sum_{K} \frac{1}{\sqrt{2 E_{M}^{f} V}}\left[a_{K}^{f} u_{M}^{f}(p) \chi_{M}^{f}(u) \exp (i \mathbf{p x})\right. \\
& \left.+b_{K}^{\dagger f} v_{M}^{f}(p) \chi_{M}^{f *}(u) \exp (-i \mathbf{p x})\right],
\end{aligned}
$$

where the index $s$ characterizes the helicity $s=\{L, R\}$ and $K=\{M, \mathbf{p}, s\} . a_{K}^{f}(t)$ is the annihilation operator for a particle in the interaction representation and $b_{K}^{\dagger f}(t)$ the antiparticle creation operator satisfying the anti-commutation rules. $E_{M}^{f}$ is the single particle energy and $\mathrm{V}$ the volume. $u_{M}^{f}(p)$ and $v_{M}^{f}(p)$ are the plane wave solutions of the Dirac equation for particles and antiparticles, respectively and the eigenfunctions $\chi_{M}(u)$ are given in (29) and (36). The general construction of states in the TB indicates that not only leptons but also scalars and gauge bosons carry the IQN $M_{A}=\left\{n, m, \varkappa, j, j_{3}\right\}$. This means that scalar fields and gauge fields can be expanded in a form analogous to (64).

The structure of the theory based on the TB geometry suggests the identification of an elementary particle as a state with specific internal quantum numbers $M$ and a specific mass analogous to quantum mechanics of atoms where discrete quantum states with different quantum numbers and energy levels exist. Therefore we do not fix the particle content from the beginning but allow for the existence of "exotic" particles which do not appear in the SM and have not been observed so far. The potential observation of such particles depends on its parameters, such as mass and lifetime, but also on selection rules as discussed below.

Inserting the expansion (64) into the fermion Laplacian (61) the Hamiltonian is easy to build with an interaction term with gauge particles $g$. For the unperturbed fermion Hamiltonian we get 
$H_{0}^{f}=\frac{1}{2} \sum_{P} \epsilon_{\mathbf{p}}\left[a_{P}^{\dagger f} a_{P}^{f}-b_{P}^{f} b_{P}^{f \dagger}\right]$,

with $P=\{M, \mathbf{p}, s\}$ and with the one-particle energy $\epsilon_{\mathbf{p}}=$ $|\mathbf{p}|$. For a compact representation we introduce for the gauge particles the notation $g=\left(g^{0}, g^{c}\right)$ with $g^{0}=\left(A, Z, E^{c}\right)$ and $g^{c}=\left(W^{ \pm}, B^{ \pm}\right)$and for the gauge potentials $A_{g \mu}=$ $A_{g \mu}^{+}+A_{g \mu}^{-}$with $A_{g \mu}^{ \pm}=a_{g \mu}^{ \pm}(x) \chi_{M_{g}}^{ \pm}(u), \chi_{M_{g}}^{+}=\left(\chi_{M_{g}}^{-}\right)^{*}$. For the interaction Hamiltonian $H_{I}^{f}$ one gets

$$
\begin{aligned}
H_{I}^{f}= & \sum_{P R K, g}\left(c_{K}^{g^{0}} V_{P R K}^{g^{0}}+c_{K}^{g^{0} \dagger} V_{P R \widetilde{K}}^{g^{0} *}+a_{K}^{g^{c}} V_{P R K}^{g^{c}}\right. \\
& \left.+b_{K}^{g^{c} \dagger} V_{P R \widetilde{K}}^{g^{c} *}\right) a_{P}^{f \dagger} a_{R}^{f}+\left(c_{K}^{g} U_{P R K}^{g}+c_{K}^{g^{\dagger}} U_{P R \widetilde{K}}^{g *}\right. \\
& \left.+a_{K}^{g^{c}} U_{P R K}^{g^{c}}+b_{K}^{g^{c} \dagger} U_{P R \widetilde{K}}^{g^{c} *}\right) b_{R}^{f} b_{P}^{f \dagger},
\end{aligned}
$$

with $R=\{N, \mathbf{r}, s\}, K=\left\{M_{g}, \mathbf{k}, \lambda\right\}, \widetilde{K}=\left\{M_{g},-\mathbf{k}, \lambda\right\}$ and

$$
\begin{aligned}
V_{P R K}^{g} & =\frac{\overline{u_{P}^{f}} \sigma_{s}^{\mu} u_{R}^{f} \epsilon_{\mu}(\mathbf{k}) I_{M N M_{g}}^{f} \delta_{\mathbf{r}, \mathbf{p}-\mathbf{k}}}{2 \sqrt{2 E_{\mathbf{p}}^{f} E_{\mathbf{r}}^{f} E_{\mathbf{k}}^{g} V}}, \\
U_{P R K}^{g} & =\frac{\overline{v_{P}^{f}} \sigma_{s}^{\mu} v_{R}^{f} \epsilon_{\mu}(\mathbf{k}) I_{M N M_{g}}^{f} \delta_{\mathbf{r}, \mathbf{p}+\mathbf{k}}}{2 \sqrt{2 E_{\mathbf{p}}^{f} E_{\mathbf{r}}^{f} E_{\mathbf{k}}^{g} V}} .
\end{aligned}
$$

Here we introduced the matrix elements

$I_{M_{g} M_{f} N_{f}}^{f}=\int \mathrm{d} \mu \chi_{M_{g}}^{g}(u) \chi_{M_{f}}^{f *}(u) \mathbf{Q}_{g} \chi_{N_{f}}^{f}(u)$,

with the integration measure $\mathrm{d} \mu(u)=\mathrm{d} \mu_{S U(2)} \mathrm{d} \mu_{E^{c}}$, $\mathrm{d} \mu_{S U(2)}=\left(16 \pi^{2}\right)^{-1} \sin \theta \mathrm{d} \theta \mathrm{d} \psi \mathrm{d} \varphi$ and $\mathrm{d} \mu_{E^{c}}=\left(4 \pi^{2}\right)^{-1}$ $\xi \mathrm{d} \xi \mathrm{d} \phi \mathrm{d} \beta$. The compact representation (66) includes all possible interactions with gauge bosons $g=A, Z, W^{ \pm}, E^{c}$ and $B^{ \pm}$.

The interaction Hamiltonian of scalar particles in the unitary gauge is described by

$$
\begin{aligned}
H_{I}^{S}= & -\sum_{P Q K R}\left(c_{P}^{S \dagger} c_{Q}^{S}+c_{\widetilde{P}}^{S} c_{\widetilde{Q}}^{S \dagger}\right) \\
& \times \Sigma_{g}\left[\left(c_{K}^{g^{0} \dagger} c_{R}^{g^{0}}+c_{\widetilde{K}}^{g^{0}} c_{\widetilde{R}}^{g^{0 \dagger}}\right) M_{P Q K R}^{g^{0}}\right. \\
& \left.+\left(a_{K}^{g^{c} \dagger} a_{R}^{g^{c}}+b_{\widetilde{K}}^{g^{c}} b_{\widetilde{R}}^{g^{c} \dagger}\right) M_{P Q K R}^{g^{c}}\right] .
\end{aligned}
$$

Here we introduced the symbols $P=\left\{M_{S}, \mathbf{p}\right\}, \widetilde{P}=$ $\left\{M_{S},-\mathbf{p}\right\}, Q=\left\{M_{S}, \mathbf{q}\right\}, \widetilde{Q}=\left\{M_{S},-\mathbf{q}\right\}, K=\left\{M_{g}, \mathbf{k}, \lambda\right\},$, $\widetilde{K}=\left\{M_{g},-\mathbf{k}, \lambda\right\}, R=\left\{M_{g}, \mathbf{r}, \sigma\right\}, \widetilde{R}=\left\{M_{g},-\mathbf{r}, \sigma\right\}$ and the matrix elements

$M_{P Q K R}^{g}=\frac{1}{8} q_{g} \frac{I_{M_{S} M_{g}}^{S} \delta_{\lambda \sigma} \delta(\mathbf{p}-\mathbf{q}+\mathbf{k}-\mathbf{r})}{V \sqrt{E_{\mathbf{p}}^{S} E_{\mathbf{q}}^{S} E_{\mathbf{r}}^{g} E_{\mathbf{k}}^{g}}}$, with $q_{A}=Q_{A}^{2}, q_{Z}=Q_{Z}^{2}, q_{E^{c}}=Q_{E^{c}}^{2}, Q_{A}=e\left(j_{3}+\frac{1}{2} m\right)$, $Q_{Z}=g \cos \theta_{D}\left(\cos ^{2} \theta_{W} j_{3}-\sin ^{2} \theta_{W} \frac{1}{2} m\right)+g_{3} \sin \theta_{D} \varkappa, q_{B}=$ $4 \varkappa\left[n+\frac{1}{2}(1+|m|)\right], q_{W}=\left[j(j+1)-j_{3}^{2}\right], Q_{E^{c}}=$ $\left(-g \cos ^{2} \theta_{W} j_{3}+g \sin ^{2} \theta_{W} \frac{1}{2} m\right) \sin \theta_{D}+g_{3} \cos \theta_{D} \varkappa$ and

$I_{M_{S} M_{g}}^{S}=\int \mathrm{d} \mu(u) \chi_{M_{S}}^{S *}(u) \chi_{M_{S}}^{S}(u) \chi_{M_{g}}^{g *}(u) \chi_{M_{g}}^{g}(u)$.

The dependence of the quantized field operators (64) on the eigenfunctions $\chi_{M}(u)$ and on the coordinates $u$ of the tangent vectors is a specific trait of the approach, here presented, based on the underlying geometric structure of the TB. The internal symmetries arise here from the inherent geometrical symmetries of the TB in an analogous way to the symmetries in quantum mechanics originating from spacetime symmetries in a given physical system. This differs in a principal way from standard QFT, therefore we call the theory here presented a tangent bundle quantum field theory.

Finally, we consider the SM Yukawa interaction $\sqrt{L_{\text {Yuk }}}$ term given by (63). Inserting the expansion (64) into (63) one gets expressions analog to the SM but including matrix elements

$I_{M_{H} M_{L} N_{R}}^{Y}=\int \mathrm{d} \mu \chi_{M_{H}}^{H}(u) \chi_{M_{L}}^{l *}(u) \chi_{N_{R}}^{l}(u)$.

The TB eigenfunctions differ for different $n$ and $\varkappa$ but from (73) we see that the Yukawa interaction is nonzero only if not only leptons and quarks but also the Higgs particles carry a nonzero $\mathrm{E}^{c}$-charge $\varkappa_{H}$. The matrix element $I_{M_{H} M_{L} N_{R}}^{Y}$ is nonzero if the relations for the hypercharges $-m_{E_{L}}+m_{\Phi}+$ $m_{E_{R}}=0$ and for the $\mathrm{E}^{c}$-charges $-\varkappa_{E_{L}}+\varkappa_{\Phi}+\varkappa_{E_{R}}=0$ are fulfilled. The solution of these equations is not unique. Since interaction processes favor the lowest magnitude of $\varkappa$ and $m$, we assume here the special solution $m=\varkappa$ for the above given two equations. For the left-handed lepton family we obtain $\varkappa_{E_{L}}=-1$, for the right-handed lepton family $\varkappa_{E_{R}}=-2$ and for the Higgs family $\varkappa_{H}=1$. An important consequence is that with $\varkappa_{H} \neq 0$ analogous as lepton families also Higgs families should exist.

\section{Lepton families, lepton universality and Higgs interaction beyond the Standard Model}

\subsection{Lepton interaction and lepton universality}

SM leptons are distinguished by the IQNs of isospin $j$ and $j_{3}$ and weak hypercharge $m$. Leptons consist of three families, electrons and electron neutrinos are members of the first family, muons and muon neutrinos of the second and taus and tau neutrinos of the third family. Different families exhibit in the SM identical IQNs and properties in the electroweak interaction with the exception of its masses. In the TB approach in addition to isospin $I$ and hypercharge $m$ the 
$E^{c}$-charge $\varkappa$ and the family quantum number $n$ exist. Here the up to now unexplained fact in the SM that three lepton families exist differentiated only by its mass find an explanation by the additional family quantum number $n$ for a nonzero $E^{c}$-charge $\varkappa$. Besides, a larger number of families than three could exist, but its possible observation depends on the mass of these states with $n \succ 3$, or possibly other physical effects.

The interaction of fermions via gauge potentials is described by (66) with analog expressions to the SM but with inclusion of the matrix elements $I_{M_{g} M_{f} N_{f}}^{f}$ given in (69) depending on the eigenfunctions of the Laplacian on the group. From (69), selection rules can be derived for fermion interactions. If the value of the integral $I_{M_{p} M_{f} N_{f}}^{f}$ is zero the interaction is forbidden. These selection rules arise in a similar way to the selection rules in atomic systems if the transition moment integral is vanishing, which constrains the possible transitions of a system from one quantum state to another. From these matrix elements we see that in the electroweak interaction leptons (with $M_{e}=N_{e}$ ) couple only to photons and Z-bosons with $\varkappa_{g}=0, j_{g}=0, m_{g}=0$ and therefore with $h_{M_{g}}(u) \equiv 1$ we find $I_{M_{A} M_{l} M_{l}}^{l}=Q$ and $I_{M_{Z} M_{l} M_{l}}^{l}=Q_{Z}$. This means that a family universal electroweak coupling of leptons with photons and Z-bosons is regained in the approach here presented . For the coupling of the families of left-handed charged leptons and neutrinos to the charged $W^{ \pm}$-bosons we substitute for $M_{l}$ the IQN of the lef-handed charged leptons and for $N_{l}$ that of the neutrinos. The operator $\mathbf{Q}_{W^{ \pm}}=g_{1} \mathbf{J}^{ \pm}$shifts the isospin component $j_{3}$ in such a way that the matrix element (69) is again independent on the family number $n$. Lepton flavor universality is one of the distinctive features of the SM and experiments have set stringent limits on processes that violate this universality. Although now every family is connected with a different IQN $n$ and different eigenfunctions, the coupling of the photons to the leptons remains independent on the family number $n$.

The same behavior we can find for the interaction with the $E^{c}$-boson with SM leptons. The matrix element (69) with $g=E^{c}$ is nonzero only if the $E^{c}$-boson carries the IQN values $\varkappa_{E^{c}}=0, j_{E^{c}}=0, m_{E^{c}}=0$.

The mass of leptons and its large difference for electrons, muons and $\tau$-leptons as well as the nonzero neutrino masses cannot be explained as a tree-level effect. But the occurrence of a family quantum number $n$ and different eigenfunctions in the approach here presented could open up a route towards its physical understanding beyond the tree level in a nonperturbative treatment. This problem is beyond the scope of the present paper.

\subsection{The interaction of Higgs particles with gauge bosons}

The interaction of scalar particles with gauge bosons is described by (70) with matrix elements given in (71) and
(72). Let us first discuss the weak interaction of the $Z$ and $W^{ \pm}$bosons with the SM Higgs particle. As explained above the $E^{c}$-charges $\varkappa$ of the $Z$ and the $W$ bosons are necessarily zero $\left(\varkappa_{Z}=0, \varkappa_{W^{ \pm}}=0\right)$. However, as described in Sect. 6 the Higgs particles carry a nonzero $\mathrm{E}^{c}$-charge (here $\varkappa_{H}=1$ is assumed), therefore the coefficients $q_{Z}$ in (71) show a very small deviation from their values in the SM proportional to $g_{3}^{2}$.

An interesting feature of the presented approach refers to the interaction of the extra gauge bosons $E^{c}$ and $B^{ \pm}$with the SM Higgs described by (70), (71) and (72). As seen the coupling of the Higgs to $E^{c}$ and $B^{ \pm}$bosons is allowed.

Note that the existence of a new vector boson is a common feature of many extensions of the SM (for a review see [37]). In particular models with an extra gauge group $U^{\prime}(1)$ are studied in a large number of papers (see e.g. [37-40]).

The possible existence of the $E^{c}$ and $B^{ \pm}$bosons leads to a fundamental fifth interaction. The parameter space of $E^{c}$ and $B^{ \pm}$masses and the coupling coefficient $g_{3}$ (or the mixing angle $\theta_{D}$ ) are constrained by existing data from experiments, and they could be found in a similar way to $U^{\prime}(1)$ extended models (see e.g. [34-36,52-56]). Many of these data hint to the assumption that the coupling coefficient $g_{3}$ and the mixing angle $\theta_{D}$ are small: $g_{3} \ll 1, \theta_{D} \ll 1$. This means that the fifth fundamental interaction mediated by the $E^{c}$ boson is much weaker than the SM weak interaction.

\section{Dark Matter candidates}

Astrophysical and cosmological observations show that the largest part of matter in our universe is constituted by unknown non-luminous particles, called DM particles, that have a very weak interaction with the visible sector of the universe. Such particles do not exist in the SM, but there have been many attempts of an extension of the SM with possible DM candidates such as weakly interacting massive particles (WIMPs), sterile neutrinos, the lightest neutralinos in super-symmetric models or axions (see e.g. $[41,42])$. Recently alternative phenomenological models have been developed, like the dark sector model (see e.g. $[36,47-51])$ or the Higgs portal model $[46,47,52,53$, $55,56]$.

One of the most notable features of the generalization of the $\mathrm{SM}$ by the gauge group $S U(2) \otimes E^{c}(2)$ is the possibility that DM candidates lie within the new gauge sector. In the present approach for the derivation of the corresponding Lagrangians of DM particles no additional phenomenological model assumptions are required, but only the particle content with the choice of appropriate IQNs for the DM is necessary. An obvious way for the assignment of the IQNs to leftand right-handed dark fermions and dark scalars can be made by the choice of zero hypercharge $(m=0)$ and isospin $(j=$ 
$0)$ but nonzero $E^{c}$-charge $\varkappa \neq 0$. As a result one may expect that, similar to the SM leptons, DM fermions and DM scalars are grouped in families with the IQN $n=1,2,3$. In the Laplacian (61), (62) and (63) we substitute for the lepton and Higgs wavefunctions $\Psi_{s}^{l}(x, u) \rightarrow \Psi_{s}^{l}(x, u)+\Psi_{s}^{D}(x, u)$ and $\Phi^{H}(x, u) \rightarrow \Phi^{H}(x, u)+\Phi^{S}(x, u)$. According to (29) the eigenfunctions of the Laplacian on the group $S U(2) \otimes E^{c}(2)$ with $j=m=0$ take the form

$$
\begin{aligned}
\chi_{M}^{D}= & h_{00 \varkappa}(\xi, \phi)=\sqrt{\frac{\varkappa}{\pi}} \exp (i \varkappa \beta) \\
& \times \exp \left(-\frac{|\varkappa| \xi^{2}}{2}\right) L_{n}^{0}\left(|\varkappa| \xi^{2}\right) .
\end{aligned}
$$

With these extensions the Laplacian (60) now is substituted by $L \rightarrow L_{\mathrm{SM}}+L_{D}$ where $L_{\mathrm{SM}}$ describe the SM particles and $L_{\mathrm{D}}=L_{\mathrm{D}}^{f}+L_{\mathrm{D}}^{S}+L_{\mathrm{D}}^{\text {Yuk }}$ includes the Lagrangians of dark fermions, dark scalars and the dark Yukawa term, respectively. In the following we discuss these Lagrangians.

\subsection{Dark vector gauge bosons}

In the present approach new vector bosons $E^{c}$ and $B^{ \pm}$arise naturally by the geometric TB symmetry described by the group $E^{c}(2)$. These particles can be interpreted as DM vector gauge bosons. The Lagrangian of the DM gauge bosons is given by (54) where the fields $C_{\mu}^{+}$and $C_{\mu}^{-}$are related with the $A_{\mu}^{c}, Z_{\mu}^{c}$ and the $E_{\mu}^{c}$ gauge potentials by the relation (52) and (57). The $E^{c}$ and $B^{ \pm}$gauge bosons are not decoupled from the SM particles, according to (62) there is a coupling to the SM Higgs, but also the interaction of the $\mathrm{E}^{c}$ boson with leptons with a very small coupling constant $g_{3}$ is allowed. Note that leptons interact also directly with the Higgs particles due to the Yukawa interaction in (63) and therefore via (62) they indirectly couple to the $E^{c}$ and $B_{\mu}^{ \pm}$bosons.

From (40), (50), (54) and (57) we can see that the nonAbelian DM vector bosons with the gauge potentials $E_{\mu}^{c}$ and $B_{\mu}^{ \pm}$interact with each other but also with the SM gauge bosons $A, Z$ and $W^{ \pm}$.

Note that the hypothesis of self-interacting DM (in contrast to collisionless cold DM) enables to resolve a number of conflicts between observations and predictions of collisionless DM simulations $[43,44]$ and has also been assumed as light thermal DM relicts [45].

\subsection{Dark fermions}

We assume that DM fermions with vanishing hypercharge and isospin $(j=0, m=0)$ but nonzero $E^{c}$-charge $(\varkappa \neq 0)$ could exist. The Lagrangian of the family of DM fermions is given by

$$
\begin{aligned}
L_{f}^{D}= & i \sum_{M s} \Psi_{M s}^{D \dagger} \sigma_{s}^{\mu} \\
& \times\left[\frac{\partial}{\partial x^{\mu}}+i g_{3} \varkappa_{D}\left(\sin \theta_{D} Z_{\mu}+\cos \theta_{D} E_{\mu}^{c}\right)\right] \Psi_{M s}^{D},
\end{aligned}
$$

with $M_{D}=\left(n, 0, \varkappa_{D}, 0,0\right)$. As one can see, different types of DM fermions with nonzero $E^{c}$-charges $\varkappa_{D}$ are predicted. For every DM fermion with given $E^{c}$-charge $\varkappa_{D}$ a DM fermion family with $n=1,2, \ldots$ could exist which couple to the SM $Z$ gauge potential with the coupling coefficient $g_{3} \varkappa_{D} \sin \theta_{D}$ and to the $E^{c}$ gauge potential with the coupling coefficient $g_{3} \varkappa_{D} \cos \theta_{D}$. Analogously to Eq. (69) one can derive corresponding selection rules for the interaction of dark fermions with gauge bosons.

The construction of a gauge and Lorentz-invariant mass term for DM fermions in a renormalizable Lagrangian can be done in a similar way to the SM using a modified Yukawa interaction term and different IQNs for right- and left-handed DM fermions. Since the SM Higgs particles carry isospin and hypercharge a DM Yukawa interaction term $L_{\text {Yuk }}^{D}$ cannot be constructed from the SM Higgs but instead a scalar DM with vanishing hypercharge and isospin $(j=0, m=0)$ but nonzero $E^{c}$-charge $\left(\varkappa_{S} \neq 0\right)$. Therefore the Yukawa interaction term for scalar DM can be expressed as

$L_{\text {Yuk }}^{D}=-\Sigma_{n_{1} n_{2}} \sigma_{n_{1} n_{2}}^{D} \Psi_{M_{D_{L}}}^{\dagger} \Phi_{M_{S}} \Psi_{N_{D_{R}}}+$ h.c.

where the sum is over the DM fermion family members with identical $\varkappa$, and $\sigma_{n_{1} n_{2}}^{D}$ are constant coupling coefficients. For the vacuum IQNs of the dark scalar we assume the IQNs $M_{S}=\left\{n_{S}=0, m_{S}=0, j_{3}=0, \varkappa_{S}=1\right\}$. This suggests that the following be assigned for the IQN values for the family of left-handed and right-handed dark fermions: $M_{D_{L}}=\left\{n_{1}, m=0, j=j_{3}=0, \varkappa_{D_{L}}=-1\right\}$, $N_{D_{R}}=\left\{n_{2}, m=0, j=0, \varkappa_{D_{R}}=-2\right\}$.

\subsection{Dark scalars}

The Lagrangian of DM scalars with $j=0, m=0$ but nonzero $x \neq 0$ and the family number $n$ is given by

$$
\begin{aligned}
L_{S}^{D}= & \partial_{\mu} \Phi_{S}^{D^{\dagger}} \partial^{\mu} \Phi_{S}^{D}+\left|\Phi_{S}^{D}\right|^{2} \\
& \times\left[g_{2}^{2} 4 \varkappa\left(n+\frac{1}{2}\right) B_{\mu}^{+} B^{\mu-}\right. \\
& +Z_{\mu} Z^{\mu}\left(g_{3} \varkappa \sin \theta_{D}\right)^{2}+E_{\mu}^{c} E^{c \mu}\left(\left(g_{3} \varkappa \cos \theta_{D}\right)^{2}\right] \\
& +V\left(\Phi_{S}^{D}, \Phi_{H}\right),
\end{aligned}
$$

where $V\left(\Phi_{S}^{D}, \Phi_{H}\right)$ is the nonlinear Higgs-type potential for the DM scalar including a possible coupling of the SM Higgs particle to the DM scalar: 


$$
\begin{aligned}
& V\left(\Phi_{S}^{D}, \Phi_{H}\right) \\
& \quad=-\mu_{S}^{2}\left|\Phi_{S}\right|^{2}+\lambda_{S}\left|\Phi_{S}\right|^{4}+\lambda_{S H}\left|\Phi_{H}\right|^{2}\left|\Phi_{S}\right|^{2} .
\end{aligned}
$$

As seen in (77) coupling of DM scalars to the DM gauge vector bosons $E^{c}$ and $B^{ \pm}$is allowed. But with a very small coefficient $g_{3} \sin \theta_{D}$ there exists also a coupling to the SM $Z$ boson arising from the diagonalization of the Laplacian for the Higgs particle. The coupling of a DM scalar particle to gauge bosons $g=\left(E^{c}, Z, B^{ \pm}\right)$is described by (70), (71) and (72) and by the eigenfunctions $\chi_{M}^{D}(u)$ as given in (74).

In order to generate the gauge boson and DM fermion mass the potential for the scalar DM should develop a nonzero VEV. Taking the extremum of $V\left(\Phi_{S}^{D}, \Phi_{H}\right)+V\left(\Phi_{H}\right)$ a nonzero VEV $\prec \Phi_{H} \succ=\Phi_{H}^{0}$ and $\prec \Phi_{S} \succ=\Phi_{S}^{0}$ can be calculated:

$$
\begin{aligned}
\left(\Phi_{H}^{0}\right)^{2} & =\frac{4 \mu_{H}^{2} \lambda_{S}-2 \mu_{S}^{2} \lambda_{S H}}{4 \lambda_{S} \lambda_{H}-\lambda_{S H}^{2}}, \\
\left(\Phi_{S}^{0}\right)^{2} & =\frac{4 \mu_{S}^{2} \lambda_{H}-2 \mu_{H}^{2} \lambda_{S H}}{4 \lambda_{S} \lambda_{H}-\lambda_{S H}^{2}} .
\end{aligned}
$$

Choosing the unitary gauge and expanding the Higgs field and the DM scalar around their VEVs by $\Phi_{H}=\Phi_{H}^{0}+\Phi_{h}$, $\Phi_{S}=\Phi_{S}^{0}+\Phi_{s}$ the mass squared matrix for the SM Higgs and for the DM scalars is given by

$M^{2}=\left[\begin{array}{ll}2 \lambda_{H}\left(\Phi_{H}^{0}\right)^{2} & \lambda_{S H} \Phi_{H}^{0} \Phi_{S}^{0} \\ \lambda_{S H} \Phi_{H}^{0} \Phi_{S}^{0} & 2 \lambda_{S}\left(\Phi_{S}^{0}\right)^{2}\end{array}\right]$.

This matrix can be diagonalized by

$$
\begin{aligned}
& \Phi_{h}^{\prime}=\cos \beta \Phi_{h}+\sin \beta \Phi_{s}, \\
& \Phi_{s}^{\prime}=-\sin \beta \Phi_{h}+\cos \beta \Phi_{s} .
\end{aligned}
$$

We have the mixing angle

$$
\tan \beta=\frac{\lambda_{S H} \Phi_{H}^{0} \Phi_{S}^{0}}{\lambda_{S}\left(\Phi_{S}^{0}\right)^{2}-\lambda_{H}\left(\Phi_{H}^{0}\right)^{2}+\Lambda}
$$

with $\Lambda=\sqrt{\left(\lambda_{S}\left(\Phi_{S}^{0}\right)^{2}-\lambda_{H}\left(\Phi_{H}^{0}\right)^{2}\right)^{2}+\left(\lambda_{S H} \Phi_{H}^{0} \Phi_{S}^{0}\right)^{2}}$. The masses for the diagonalized mass eigenstates are

$M_{h^{\prime}}=\lambda_{S}\left(\Phi_{S}^{0}\right)^{2}+\lambda_{H}\left(\Phi_{H}^{0}\right)^{2}+\Lambda$,

$M_{s^{\prime}}=\lambda_{S}\left(\Phi_{S}^{0}\right)^{2}+\lambda_{H}\left(\Phi_{H}^{0}\right)^{2}-\Delta$.

Spontaneous symmetry breaking of the SM Higgs field and the DM scalar leads to the generation of masses for the gauge bosons $Z, W^{ \pm}$as well as for the dark vector bosons $E^{c}$ and $B^{ \pm}$which contain contributions from the Higgs VEV as well as from the DM scalar VEV,

$$
\begin{aligned}
M_{W^{ \pm}}^{2}= & \left(\Phi_{H}^{0}\right)^{2} g_{1}^{2} / 2 \\
M_{Z}^{2}= & 2\left(\Phi_{H}^{0}\right)^{2}\left[\frac{1}{2} g \cos \theta_{D}-g_{3} \sin \theta_{D}\right]^{2} \\
& +2\left(g_{3} \varkappa \sin \theta_{D}\right)^{2}\left(\Phi_{S}^{0}\right)^{2},
\end{aligned}
$$

$$
\begin{aligned}
M_{E^{c}}^{2}= & 2\left(\Phi_{H}^{0}\right)^{2}\left[\frac{1}{2} g \sin \theta_{D}+g_{3} \cos \theta_{D}\right]^{2} \\
& +2\left(g_{3} \varkappa \cos \theta_{D}\right)^{2}\left(\Phi_{S}^{0}\right)^{2}, \\
M_{B^{ \pm}}^{2}= & 2 g_{2}^{2}\left[2\left(\Phi_{H}^{0}\right)^{2}+\left(\Phi_{S}^{0}\right)^{2}\right] .
\end{aligned}
$$

The mass of the $W^{ \pm}$bosons is identical to its value in the SM. For the Z-boson we obtain from (85) a mass with a small deviation from the SM proportional to $\left(g_{3}\right)^{2}$ for $g_{3} \ll 1: \delta M_{Z}=\left(\Phi_{H}^{0}\right) 4 g_{3}^{2} / \sqrt{2} g$, which can be used for the determination of experimental bounds of the coupling coefficient $g_{3}$. As seen in (87) with $\varkappa_{H}=1, m_{H}=1, n_{H}=0$, the $B^{ \pm}$boson gets a mass proportional to the SM coupling constant $g_{2}$. Without the VEV of the dark scalar (with $\Phi_{S}^{0} \rightarrow 0$ ) one gets $M_{B^{ \pm}}=\Phi_{H}^{0} g_{2} 2 \simeq 116.24 \mathrm{GeV}$ (using $\Phi_{H}^{0}=180$ $\mathrm{GeV}$ ). According to (86) the mass of the $\mathrm{E}^{c}$ boson given by $M_{E^{c}} \simeq 2 \Phi_{S}^{0} g_{3}$ for $g_{3} \ll 1$ is proportional to $g_{3}$.

Albeit on a different theoretical basis, the described predictions show some similar features that arise in various scenarios for DM physics denoted as the dark sector (see e.g. [36,47-51]), the vector Higgs portal (see e.g. $[46,47,52,53,55,56]$ and the Z-portal (see e.g. [54,55]). The dark sector hypothesis assumes that DM interacts only through a new $U_{D}(1)$ force with a hypothetical "dark photon" as gauge boson but SM matter does not interact directly with DM particles but can interact indirectly via a kinetic mixing term in the Lagrangian [48]. In the Higgs portal model a DM massive vector boson associated with a hidden U' (1) symmetry couples to the SM Higgs and in the Zportal DM model a DM fermion interacts directly with the SM Z-boson. Reference [55,56] reports that the Higgs portal model is compatible with the available data. Besides in [55] also acceptable regions of parameters for the Z-portal model for the interaction of DM fermions with the SM Z-boson were reported. From these results similar conclusions can be drawn for the acceptable parameter region of the Lagrangians (75) and (77).

A comparison of the approach here presented with the Higgs portal and the Z-portal models shows some common properties but also clearly distinct features and principal differences. Whereas the majority of DM models can be considered as a minimal extension of the SM based on a phenomenological model assumption to understand the mechanism of annihilation, scattering and possible decays of SM particles the idea of the present approach is to describe fundamental interactions of SM particles as well as DM particles in a uniform way without phenomenological model assumption within the geometrical structure of the TB with the same symmetry group $S U(2) \otimes E^{c}(2)$. As seen in the Lagrangian (62) the SM Higgs particle interacts with the dark vector bosons $E^{c}$ and $B^{ \pm}$, and in the Lagrangian (77) a DM scalar and in (75) a DM fermion with the SM $Z$ boson. The extra $\mathrm{DM}$ vector bosons $E^{c}$ and $B^{ \pm}$are nonabelian gauge 
bosons and interact with the SM gauge bosons. Due to the existence of families the SM leptons carry an $E^{c}$-charge. A nonzero Yukawa interaction term hints that also the Higgs particle carries a nonzero $E^{c}$-charge $\varkappa$ and therefore a family of Higgs particles could exist. The coupling of the Higgs and DM scalars in (62) and (77) with the $E^{c}$ and $B^{ \pm}$arise in an intrinsic way by the symmetry group, while interactions of the Z-Boson with the DM $E^{c}$ and $B^{ \pm}$boson or a scalar DM particle arise due to the diagonalization of the Higgs Lagrangian by the transformations (57).

Above we discussed only DM candidates with zero isospin and hypercharge, but there exists the possibility that DM particles basically interact by the weak coupling to SM particles. This means they are electrically neutral but carry hypercharge and isospin satisfying the relation $Q_{A}=e\left(j_{3}+\frac{1}{2} m\right)=0$. This includes right-handed sterile neutrinos with $j_{3}=\frac{1}{2}$ and $m=-1$.

\section{Conclusions}

The present paper is based on the hypothesis that the tangent bundle is the underlying geometrical structure for the description of the fundamental physical interactions. The internal (gauge) symmetries are not inserted as an extra theory constituent given externally a priori by phenomenological reasons like in the SM but emerge from the inherent geometrical structure of the TB with symmetries described by the group $S O(3,1)$. Projective irreducible representations of this group can be constructed by using the little groups $S U(2), E^{c}(2)$ and $S U(1,1)$. Using the covariant derivative given by the operators on the transformation group $G=S U(2) \otimes E^{c}(2)$, and the corresponding connection coefficients (gauge potentials) a generalized theory of the electroweak interaction is derived. Since the SM arises (without phenomenological assumptions) as a limit case the presented approach answers the question why the gauge group of the electroweak interaction in the SM is $G=S U(2) \otimes U(1)$.

In the TB approach wave functions depend on the spacetime coordinates $x$ as well as on the coordinates of the tangent fibers $u$. The well-known SM $Z$ and $W^{ \pm}$gauge bosons can be found again but in addition new extra gauge bosons $E^{c}$ and $B^{ \pm}$are predicted which constitute a fifth fundamental interaction. In addition to the SM quantum numbers of isospin and hypercharge, the $E^{c}$-charge $\varkappa$ and the family quantum number $n$ exist. The existence of the family IQN $n$ in the TB approach sheds light on the mysterious appearance of lepton families in the SM requiring a distinct IQN for every family. However, the large mass difference of different families cannot be explained as a tree-level effect but requires a non-perturbative quantum loop treatment which is beyond the scope of the present paper. The existence of families requires that leptons carry a nonzero $E^{c}$-charge $\varkappa_{l}$. A selection rule from the Yukawa interaction indicates that also the Higgs particle carries the $E^{c}$-charge $\varkappa_{H}=1$ and therefore in the present approach a family of Higgs particles is predicted. In contrast, $\mathrm{SM}$ and $E^{c}$ gauge bosons carry zero $E^{c}$-charge, $\varkappa_{g}=0$. The derived selection rule reveals that a family universal coupling of leptons persists for the interaction with the $S M$ gauge bosons.

An important prediction of the theory presented is the possibility of identifying candidate stable or unstable hypothetical DM fermions and DM scalars with zero hypercharge and zero isospin but nonzero $E^{c}$-charge $\varkappa \neq 0$ which should necessarily be grouped in different families with different family numbers $n=1,2, \ldots$ The new non-Abelian vector bosons $E^{c}$ and $B^{ \pm}$can be interpreted as DM vector gauge bosons. These hypothetical bosons are not decoupled from the SM particles, but there is a weak coupling to the SM Higgs and to the SM Z-boson. Moreover, also the coupling of leptons with the $E^{c}$ bosons with a small coupling coefficient $g_{3}$ is allowed. DM vector bosons interact with each other but also with the SM gauge bosons. Spontaneous symmetry breaking of the SM Higgs and the DM scalar predicts not only the masses of the $Z$ and $W^{ \pm}$bosons but also that of the dark gauge bosons $E^{c}$ and $B^{ \pm}$. The precondition of nonzero $E^{c}$-charges $\varkappa \neq 0$ of DM fermions and DM scalars indicates that analogous to lepton families also DM fermion and DM scalar families should exist.

Finally, the approach presented is linked with the geometrization program of physics based on the single hypothetical principle that the tangent bundle with the symmetry group $S O(3,1) \rtimes T(3,1)$ is the fundamental geometrical structure for an unified description of all fundamental physical interactions. On the one hand as briefly explained in Sect. 3 the tangent bundle is the geometrical fundament for teleparallel gravity gauge theory based on translational transformations $T(3,1)$ of tangent vectors along the fiber axis $[11-$ 15] which is fully equivalent to the Einstein gravity theory. On the other hand here a generalized theory of electroweak interaction and dark matter is presented based on the little groups of $S O(3,1)$. Therefore gravity, electroweak interaction and DM are described by the same fundamental geometrical structure of the TB. Note that strong interaction with the gauge group $S U(3)$ is in this frame still missing. The color group $S U$ (3) of quantum chromodynamics cannot be described as a geometrical symmetry in the TB in the same way as the $S U(2) \otimes E^{c}(2)$ group leaving the scalar product (3) invariant. However, the $S U$ (3) symmetry could be hidden in the fundamental of the tangent bundle geometry in a surprising way arising as an emergent symmetry similar to Chern-Simon gauge fields that originate by the anomalous quantum Hall effect in solid state theory (see e.g. [57-60]. The key for this assumption is the fact that the eigenfunction of the $E^{c}(2)$ group given in (29) has the same form as 
the solution of the 2D Schrödiner equation for electrons in a perpendicular external magnetic field. The solution (29) describes the vertical subspace for a single tangent fiber at a fixed spacetime point, but if we combine all tangent fibers at all spacetime points we get an equation with an analog form to the multi-particle Schrödinger equation of a $2 \mathrm{D}$ quantum Hall system and, with the account of the three iso-spin components of fermions, that of a three-layer quantum Hall system [58]. This explains the astonishing analogy of fractional charge quantization of quarks with the anomalous qantum Hall effect [57]. In this approach emergent effective fields $[58,59,61,62]$ (arising here in the vertical subspace of tangent vectors in the TB) originate in an intrinsic way and bound the bare quarks to two vortices constituting composite quarks. These gauge fields and the SU(3) symmetry are assigned to the base spacetime of the TB via the density distributions and could appear as the local SU(3) color symmetry of quantum chromodynamics.

Data Availability Statement This manuscript has no associated data or the data will not be deposited. [Authors' comment: This is a theoretical study and no experimental data has been listed.]

Open Access This article is distributed under the terms of the Creative Commons Attribution 4.0 International License (http://creativecomm ons.org/licenses/by/4.0/), which permits unrestricted use, distribution, and reproduction in any medium, provided you give appropriate credit to the original author(s) and the source, provide a link to the Creative Commons license, and indicate if changes were made. Funded by SCOAP ${ }^{3}$.

\section{References}

1. E. Lubkin, Ann. Phys. (N.Y.) 23, 233 (1963)

2. A. Trautman, Rep. Math. Phys. 1, 29 (1970)

3. W. Drechsler, M.E. Meyer, Fibre Bundle Techniques in Gauge Theories, Lecture Notes in Physics (Springer, 1977)

4. M. Daniel, C.M. Viallet, Rev. Mod. Phys. 52, 175 (1980)

5. M. Nakahara, Geometry, Topology and Physics (Institute of Physics, Bristo, 1990)

6. B.-Y. Hou, Differential Geometry for Physicists (Singapore, 1997)

7. S. Coleman, J. Mandula, Phys. Rev. 189, 1251 (1967)

8. S. Kobayashi, K. Nomizu, Foundation of Differential Geometry (Interscience Publishers, Geneva, 1963)

9. Chris J. Isham, Modern Differential Geometry for Physicists (World Scientific, Singapore, 1999)

10. J.A. Schouten, Ricci Calculus (Springer, Berlin, 1954)

11. K. Hayashi, T. Nakno, Prog. Theor. Phys. 38, 491 (1967)

12. Y.M. Cho, Phys. Rev. D 14, 2521 (1976)

13. T. Dass, Pramana 23, 433 (1984)

14. R. Aldrovandi, J.G. Pereira, Teleparallel Gravity; An Introduction (Springer, Dordrecht, 2012)

15. J.W. Maluf, Ann. Phys. 525, 339 (2013). arXiv:1303.3897 [gr-qc]

16. T.W. Kibble, J. Math. Phys. 2, 212 (1961)
17. F.W. Hehl, J.D. McCrea, E.W. Mielke, Y. Ne'eman, Phys. Rep. 258, 1 (1995)

18. F.W. Hehl, J.D. McCrea, E.W. Mielke, Y. Ne'eman, Phys. Rev. D 48, 48 (1993)

19. E.P. Wigner, Ann. Math. 40, 149 (1939)

20. V. Bargman, Ann. Math. 59, 1 (1954)

21. H. Hoogland, J. Phys. A Math. Gen. 11, 1557 (1978)

22. J.F. Carina, M.A. del Olmo, M. Santander, J. Phys. A Math. Gen. 17, 3091 (1984)

23. J.J. Quinn, A. Wójs, K.-S. Yi, G. Simion, Phys. Rep. 481, 29 (2009)

24. V. Aldaya, J. Navarro-Salas, J. Bisquert, R. Loll, J. Math. Phys. 33, 3087 (1992)

25. I.M. Shapiro, R.A. Minlos, Z. Ya Shapiro, Representations of the Rotation and Lorentz, Groups and their Applications (Pergamon Press, Oxford, 1963)

26. W.J. Holman, L.C. Biedenhan Jr., Ann. Phys. 39, 1 (1966)

27. D. Cangemi, R. Jackiw, Phys. Rev. Lett. 69, 233 (1992)

28. C. Nappi, E. Witten, Phys. Lett. B 293, 309 (1992)

29. A. Tseytlin, Nucl. Phys. B 450, 231 (1995)

30. K. Sfetsos, Phys. Lett. B 324, 335 (1994)

31. P.A. Grassi, Nucl. Phys. B 560, 499 (1999)

32. F. Ferrari, J. Math. Phys. 44, 138 (2003)

33. F.R. Ruiz, Eur. Phys. J. C 76, 94 (2016)

34. R. Essig et al., JHEP 02, 009 (2011)

35. J.D. Bjorken et al., Phys. Rev. D 80, 07501 (2009)

36. J. Alexander et al., Dark sectors 2016 workshop: community report, ArXiv: $1608.08632 \mathrm{v} 1$

37. P. Langacker, Rev. Mod. Phys. 81, 1199 (2009)

38. T. Appelquist, B.A. Dobrescu, A.R. Hopper, Phys. Rev. D 68, $035012(2003)$

39. K.S. Babu, C. Kolda, J. March-Russell, Phys. Rev. D 57, 6788 (1998)

40. F. Blas, M. Perez-Victoria, F. del Aguila, J. de Blas, M. PerezVictoria, JHEP 1009, 033 (2010)

41. G. Bertone, D. Hooper, J. Silk, Particle dark matter: evidence, candidates and constraints. Phys. Rep. 405, 279 (2005)

42. Stefan Profumo, An Introduction to Particle Dark Matter (World Scientific, Singapore, 2017)

43. D.N. Steinhardt, J. Paul, Phys. Rev. Lett. 84, 3760 (2000)

44. S. Tulin, H.-B. Yu, Phys. Rep. 730, 1 (2018)

45. Y. Hochberg et al., Phys. Rev. Lett. 113, 171301 (2014)

46. V. Silveira, A. Zee, Phys. Lett. B 161, 136 (1985)

47. Thomas Hambye, JHEP 01, 028 (2009)

48. B. Holdom, Phys. Lett. B 166, 196 (1986)

49. P.-F. Yin, S.-H. Zhu, Front. Phys. 11, 111403 (2016)

50. N. Bernal et al., JCAP 1603, 028 (2016)

51. S.-M. Choi et al., JHEP 1710, 162 (2017)

52. S. Kanemura et al., Phys. Rev. D 82, 055026 (2010)

53. O. Lebedev, H. M:Lee, Y. Mambrini, Phys. Lett. B 702, 570 (2012)

54. G. Arcadi, Y. Mambrini, F. Richard, arXiv:1411.2985v1

55. J. Ellis, A. Fowlie, L. Marzola, M. Raidal, Phys. Rev. D 97, 115014 (2018)

56. P. Athron et al., Eur. Phys. J. C 79, 38 (2019)

57. R.B. Laughlin, Phys. Rev. Lett. 50, 1395 (1983)

58. Z.F. Ezawa, Quantum Hall Effects (World Scientific, Singapore, 2013)

59. O. Heinonen (ed.), Composite Fermions (World Scientific, Singapore, 1998)

60. J.K. Jain, Composite Fermions (Cambridge University Press, Cambridge, 2009)

61. S.C. Zhang, H. Hansson, S. Kivelson, Phys. Rev. Lett. 62, 82 (1989)

62. A. Lopez, E. Fradkin, Phys. Rev. B 44, 5246 (1995) 